CRYSTALLOGRAPHIC COMMUNICATIONS

ISSN 2056-9890

Received 3 December 2018

Accepted 15 December 2018

Edited by W. T. A. Harrison, University of Aberdeen, Scotland

Keywords: crystal structure; quinoxaline cavitands; inclusion compounds; benzene.

CCDC reference: 1885516

Supporting information: this article has supporting information at journals.iucr.org/e

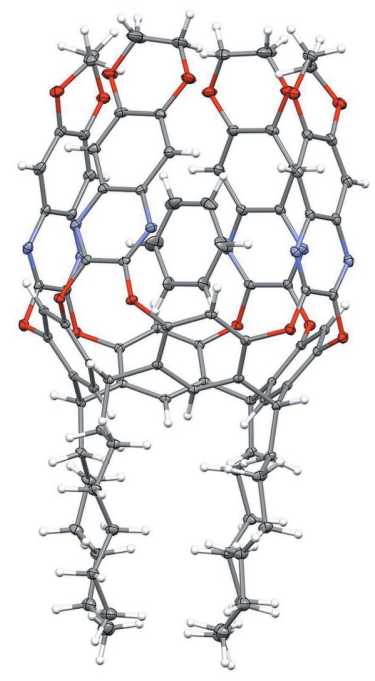

OPEN $\odot$ ACCESS

\section{A new, deep quinoxaline-based cavitand receptor for the complexation of benzene}

\author{
Roberta Pinalli, Jakub W. Trzciński, Enrico Dalcanale and Chiara Massera* \\ Dipartimento di Scienze Chimiche, della Vita e della Sostenibilità Ambientale, Università di Parma, Parco Area delle \\ Scienze 17/A, 43124 Parma, Italy. *Correspondence e-mail: chiara.massera@unipr.it
}

We report the synthesis of a new macrocyclic receptor, namely 2,8,14,20tetrahexyl-4,24:6,10:12,16:18,22- $O, O^{\prime}$-tetrakis[2,3-dihydro-[1,4]dioxino[2,3-g]quinoxalin-7,8-diyl]resorcin[4]arene, DeepQxCav, obtained by the addition of ethylene glycol ditosylate to an octahydroxy quinoxaline cavitand. A 1:1 supramolecular complex of this cavitand with benzene has been obtained and analysed through X-ray diffraction analysis. The complex, of general formula $\mathrm{C}_{92} \mathrm{H}_{88} \mathrm{O}_{16} \mathrm{~N}_{8} \cdot \mathrm{C}_{6} \mathrm{H}_{6}$, crystallizes in the space group $C 2 / c$, with the cavitand host located about a twofold rotation axis. The benzene guest, which is held inside the cavity by $\mathrm{C}-\mathrm{H} \cdots \pi$ interactions and dispersion forces, is disordered over two equivalent sites, one in a general position and one lying on a twofold axis. The crystal structure features $\mathrm{C}-\mathrm{H} \cdots \mathrm{O}$ hydrogen bonds and $\mathrm{C}-\mathrm{H} \cdots \pi$ interactions involving the alkyl chains, the aromatic rings, and the $\mathrm{O}$ atoms of the dioxane moiety of the resorcinarene scaffold. The crystal studied was refined as a twocomponent twin.

\section{Chemical context}

Resorcinarene-based cavitands are macrocyclic synthetic compounds (Cram, 1983; Cram \& Cram, 1994), whose versatility primarily stems from the possibility of modifying the size and form of the cavity by choosing different bridging groups connecting the phenolic hydroxyl groups of the resorcinarene scaffold. This allows the tuning of the complexation properties of the cavity, which can thus interact with neutral and charged molecules through hydrogen bonding, $\pi-\pi$ stacking and $\mathrm{C}-$ $\mathrm{H} \cdots \pi$ interactions, but also forms coordinate bonds with metal centers to create discrete complexes, cages or extended networks. These properties have made cavitands useful receptors for molecular recognition and building blocks for crystal engineering (Pinalli et al., 2016; Kane et al., 2015; Brekalo et al., 2018). In our group, we have been exploiting two main types of receptors, in which the bridging groups at the upper rim are either phosphonate $R \mathrm{PO}_{3}$ moieties or quinoxaline ring systems. Both families have been extensively used in sensing in solution (Lee et al., 2018; Liu et al. 2018) and in the gas phase (Melegari et al., 2013; Tudisco et al., 2016). Indeed, the demand for fast and reliable detection of biological and chemical hazards is rising continuously and optimal sensors for environmental, security and biomedical applications must be sufficiently responsive to allow detection of the target analyte at low concentrations, and selective enough to respond primarily to a single chemical species in the presence of interferents. In this respect, quinoxaline-based cavitands, exploiting the $\pi$-basicity and hydrophobicity of their cavity are ideal hosts to interact with aromatic compounds (Pinalli et al., 2018). Following this line of 
research, we have synthesized a new member of the quinoxaline family, DeepQxCav, in which the cavity has been made deeper by the addition of four 1,4 dioxane rings on the quinoxaline walls. In this paper we report and analyse the crystal structure of its complex with benzene as guest.

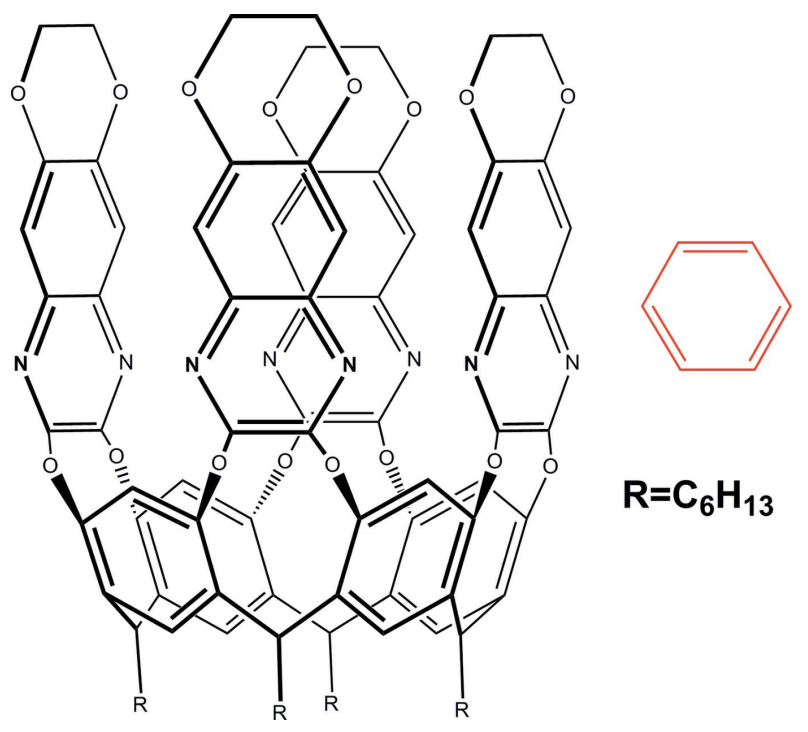

\section{Structural commentary}

The molecular structure of DeepQxCav is shown in Fig. 1. It consists of a 1:1 host-guest complex in which one molecule of benzene is engulfed inside the walls of the cavitand. The complex crystallizes in the monoclinic crystalline system, in space group $C 2 / c$. The asymmetric unit comprises half of a cavitand host in a general position, about a twofold rotation axis, and half a molecule of the benzene guest disordered over
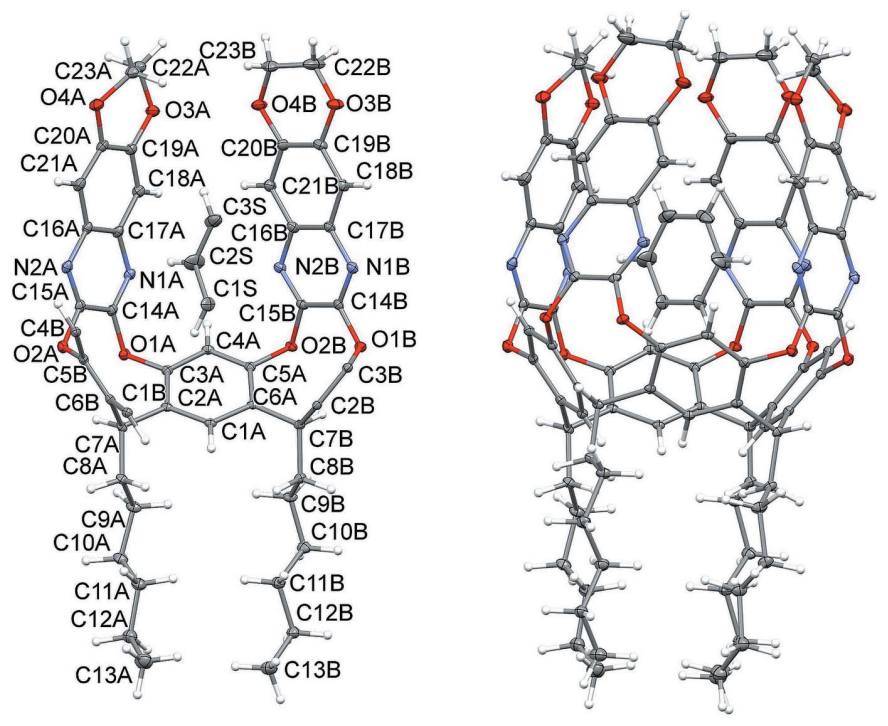

Figure 1

Left: asymmetric unit of the title compound with labelling scheme and ellipsoids drawn at the $20 \%$ probability level. Right: molecular structure of the whole complex. The symmetry-related atoms are in position $1-x$, $y, \frac{1}{2}-z$. For both views, only one of the two disordered orientations has been shown for clarity.
Table 1

Selected interatomic distances $(\AA)$.

\begin{tabular}{llll}
\hline $\mathrm{C} 22 A \cdots \mathrm{C} 23 B$ & $4.053(9)$ & $\mathrm{C} 22 A \cdots \mathrm{C} 23 A^{\mathrm{i}}$ & $8.181(4)$ \\
$\mathrm{C} 23 A \cdots \mathrm{C} 22 B^{\mathrm{i}}$ & $3.757(5)$ & $\mathrm{C} 22 B \cdots \mathrm{C} 23 B^{\mathrm{i}}$ & $4.664(9)$ \\
\hline
\end{tabular}

Symmetry code: (i) $-x+1, y,-z+\frac{1}{2}$.

two sites, one in a general position and one lying on a twofold axis. Distances and angles are in good agreement with similar compounds reported in the literature (see Section 4).

Fig. 2 shows two perspective views of the shape of the cavity (in a vase conformation) upon complexation of the guest. The depth of the cavity has been calculated as the distance between the mean plane passing through the groups of atoms $\mathrm{C} 7$ at the lower rim and $\mathrm{C} 22-\mathrm{C} 23$ of the upper rim, yielding a value of 10.290 (2) $\AA$. The mean planes passing through the quinoxaline moieties are inclined with respect to the plane passing through the $\mathrm{O} 1 / \mathrm{O} 2$ atoms (see Fig. 2), forming angles of 85.24 (3) and $75.16(4)^{\circ}$ for the walls labelled $A$ and $B$, respectively. The mouth of the cavity is roughly rectangular in shape, but because of the bending of the walls, the opening is blocked by the steric hindrance of the four 1,4 dioxane rings (see Table 1 for geometrical details).

\section{Supramolecular features}

The most interesting supramolecular feature of the title compound is the encapsulation of benzene inside the aromatic cavity of the host. As can be seen in Fig. 3, two orientations of the guest are present, from now on called I for ring $\mathrm{C} 1 S-\mathrm{C} 3 S$ and II for ring $\mathrm{C} 4 S-\mathrm{C} 7 S$ (the remaining atoms are generated by symmetry). I and II are rotated by $c a 60^{\circ}$ with respect to each other. In both cases, the benzene molecule is found deep inside the cavity, at the same level of the pyrazine rings, roughly parallel to the walls labelled $B$ [the angles formed by the mean planes passing through the benzene rings and through the quinoxaline wall are $82.4(5)$ and $84.2(3)^{\circ}$ for I and II, respectively] and perpendicular to the walls labelled $A$ [angles of 15.3 (2) and 15.0 (2) ${ }^{\circ}$ for I and II, respectively]. In particular, the distances of $\mathrm{C} 1 S$ and $\mathrm{C} 7 \mathrm{~S}$ from the mean plane passing through the resorcinarene oxygen atoms $\mathrm{O} 1 / \mathrm{O} 2$ are
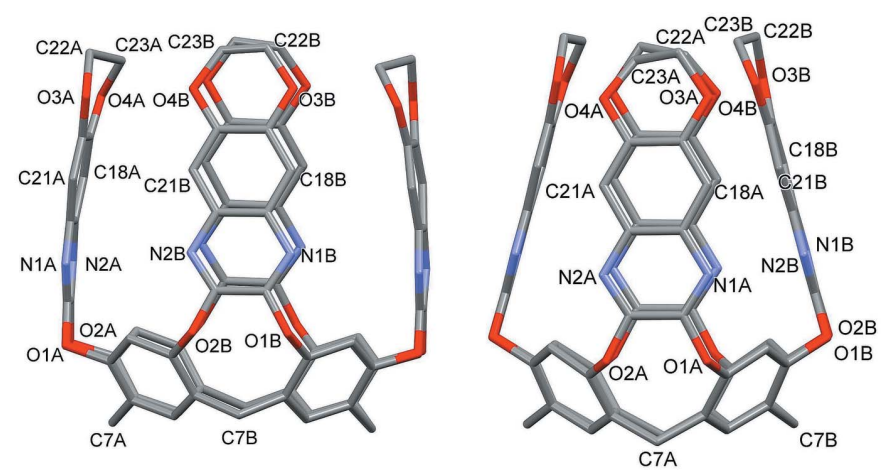

Figure 2

Perspective views of the cavity of DeepQxCav with partial labelling scheme, referred only to atoms in general positions. $\mathrm{H}$ atoms and alkyl chains have been omitted for clarity. 
Table 2

Hydrogen-bond geometry $\left(\AA,^{\circ}\right)$.

$\mathrm{Cg} 1$ is the centroid of the ring $\mathrm{C} 1 B-\mathrm{C} 6 B ; \mathrm{Cg} 2$ is the centroid of the ring $\mathrm{C} 14 A-$ $\mathrm{C} 17 A / \mathrm{N} 1 A / \mathrm{N} 2 A$.

\begin{tabular}{lllll}
\hline$D-\mathrm{H} \cdots A$ & $D-\mathrm{H}$ & $\mathrm{H} \cdots A$ & $D \cdots A$ & $D-\mathrm{H} \cdots A$ \\
\hline $\mathrm{C} 21 B-\mathrm{H} 21 B \cdots \mathrm{O} 3 A^{\mathrm{ii}}$ & 0.95 & 2.50 & $3.307(3)$ & 143 \\
$\mathrm{C} 18 A-\mathrm{H} 18 A \cdots \mathrm{O} 3 A^{\mathrm{ii}}$ & 0.95 & 2.40 & $3.302(2)$ & 158 \\
$\mathrm{C} 8 B^{\mathrm{iii}}-\mathrm{H} 8 B 2^{\mathrm{iii}} \cdots \mathrm{C} 20 A$ & 0.99 & 2.71 & $3.693(3)$ & 170 \\
$\mathrm{C} 23 A-\mathrm{H} 23 A \cdots \mathrm{Cg} 1^{\mathrm{iv}}$ & 0.99 & 2.88 & $3.530(4)$ & 124 \\
$\mathrm{C} 12 B^{\mathrm{v}}-\mathrm{H} 12 D^{\mathrm{v}} \cdots \mathrm{O} 1 A$ & 0.99 & 2.71 & $3.414(3)$ & 128 \\
$\mathrm{C} 2 S-\mathrm{H} 2 S \cdots \mathrm{C} 22^{\mathrm{i}}$ & 0.95 & 2.67 & $3.609(3)$ & 171 \\
\hline
\end{tabular}

Symmetry codes: (i) $-x+1, y,-z+\frac{1}{2}$; (ii) $-x+1,-y+2,-z+1$; (iii) $x+\frac{1}{2}, y+\frac{1}{2}, z$; (iv) $-x+\frac{3}{2}, y+\frac{1}{2},-z+\frac{3}{2} ;$ (v) $-x+1,-y+1,-z+1$.

1.128 (5) and 1.003 (9) $\AA$, respectively. In the case of orientation I, two symmetry-related, equivalent $\mathrm{C}-\mathrm{H} \cdots \pi$ interactions are present, between $\mathrm{C} 2 \mathrm{~S}-\mathrm{H} 2 \mathrm{~S}$ and the centroid $\mathrm{Cg} 2$ of the ring (see Table 2). These interactions are absent in the case of orientation II, which is stabilized by van der Waals dispersion forces only.

In the crystal, the main interactions connecting the cavitands are $\mathrm{C}-\mathrm{H} \cdots \mathrm{O}$ hydrogen bonds, involving the $\mathrm{C}-\mathrm{H}$ groups of the alkyl chains $(\mathrm{C} 12 B-\mathrm{H} 12 D)$ and of the aromatic rings $(\mathrm{C} 18 A-\mathrm{H} 18 A, \mathrm{C} 21 B-\mathrm{H} 21 B)$ with the oxygen atoms of the dioxane moiety $(\mathrm{O} 3 A)$ or of the resorcinarene scaffold (O1A, see Figs. 4 and 5 and Table 2). Further consolidation of the structure is provided by $\mathrm{C}-\mathrm{H} \cdots \pi$ interactions (Fig. 4) due to the presence of aromatic rings in the cavitand scaffold.

\section{Database survey}

Several structures of quinoxaline-based cavitands have been published in recent years. A search in the Cambridge Structural Database (Version 5.38, update August 2018; Groom et al., 2016) yielded 20 hits, of which 15 were inclusion compounds. In particular, the group of Professor Dalcanale has reported compounds NUTBUB01 and GURLIQ (Bertani et al., 2016) in which a singly or doubly 'roofed' quinoxaline cavitand forms a 1:1 complex with benzene; LIMFOE and LIMGAR (Pinalli et al., 2013) in which the guests are 1,3benzodioxole and 5-allyl-1,3-benzodioxole, respectively; and
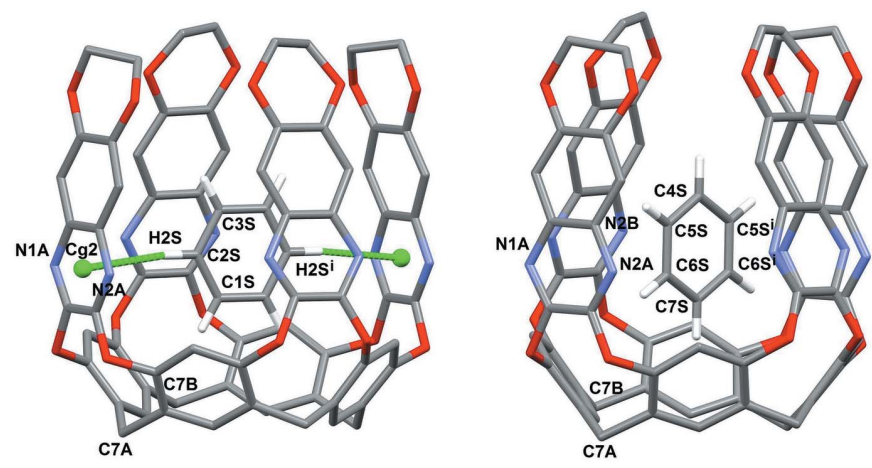

Figure 3

View of the interactions (green dotted lines) involving the benzene ring and the quinoxaline cavitand (both orientations of the guest are shown). Symmetry code: (i) $1-x, y, \frac{1}{2}-z$.

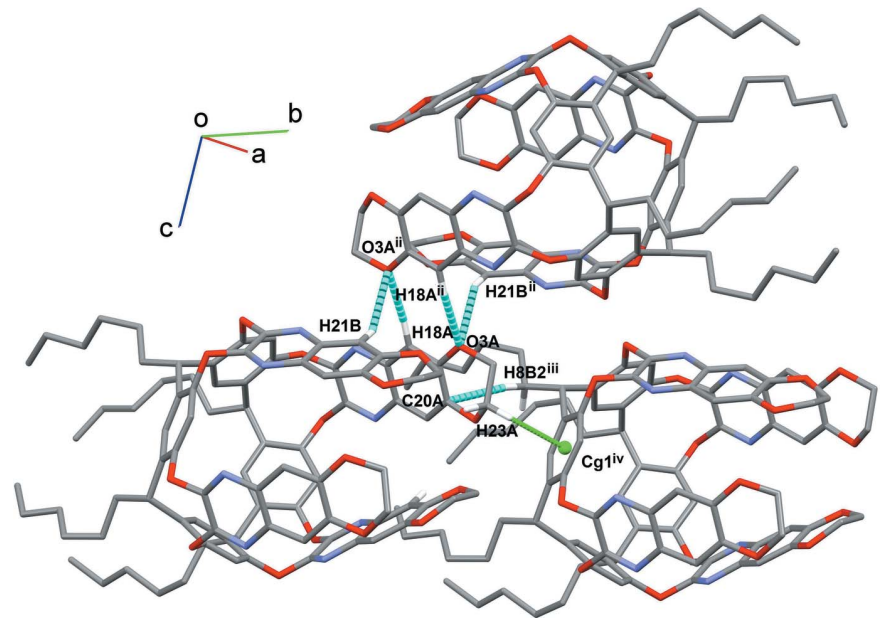

Figure 4

View of the relevant $\mathrm{C}-\mathrm{H} \cdots \mathrm{O}$ hydrogen bonds and $\mathrm{C}-\mathrm{H} \cdots \pi$ interactions (light-blue and green dotted lines, respectively) stabilizing the crystal structure of the title compound. Only the $\mathrm{H}$ atoms involved in the interactions are shown. The benzene guest has been omitted for clarity.

the fluorobenzene complex YAGVIL (Soncini et al., 1992). Other related compounds are BUJNUR (Ballistreri et al., 2016), a benzene clathrate co-crystallizing with fullerene; LUDJEA (Wagner et al., 2009), in which the guest is phenyl azide; and UNIFAY (Azov et al., 2003), an inclusion compound with acetonitrile, the only non-aromatic guest of the series.

Particularly interesting is a quinoxaline-based cavitand (EtQxBox) in which four ethylendioxy bridges between the quinoxaline wings have been introduced to obtain a rigidification of the cavity (Trzciński et al., 2017). Also in that case, the crystal structure of the inclusion compound with benzene has been obtained and analysed in detail in the solid state.

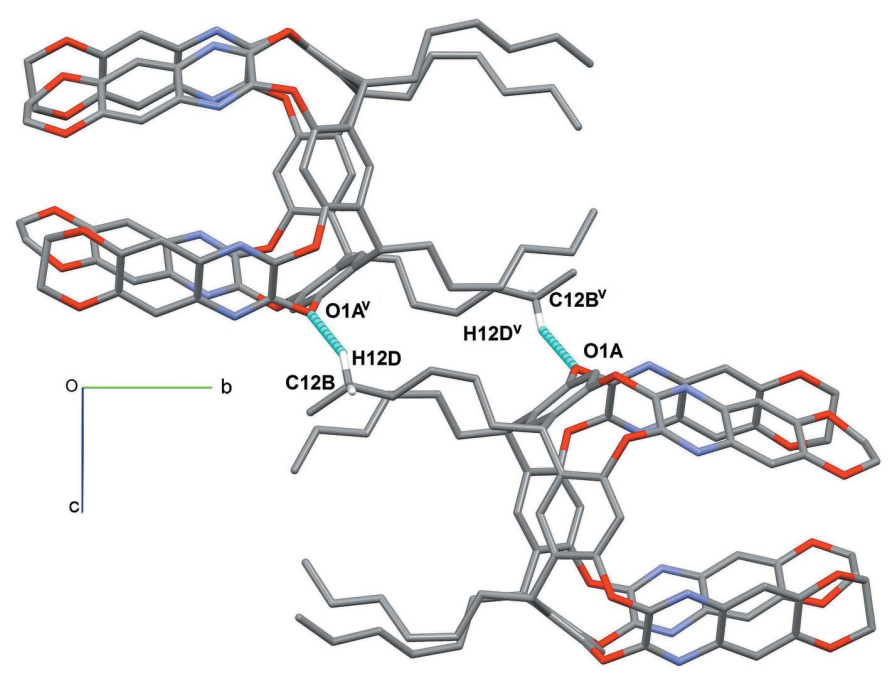

Figure 5

View of the relevant $\mathrm{C}-\mathrm{H} \cdots \mathrm{O}$ hydrogen bonds (light-blue dotted lines) stabilizing the crystal structure of the title compound. Only the $\mathrm{H}$ atoms involved in the interactions are shown. The benzene guest has been omitted for clarity. 


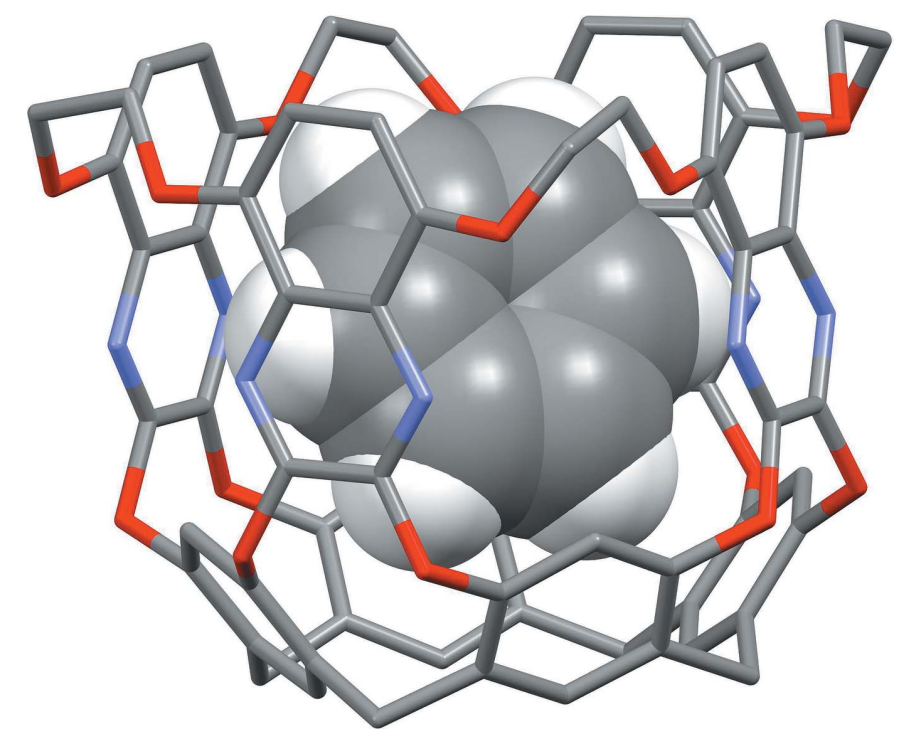

Figure 6

View of the orientation of benzene (in space-filling mode) inside the rigidified cavitand EtQxBox. Alkyl chains and host $\mathrm{H}$ atoms have been omitted for clarity.

Differently from what happens in the title compound, the benzene molecule does not lie parallel to the quinoxaline walls of EtQxBox (Fig. 6) and is held inside the cavity by two C$\mathrm{H} \cdots \pi$ interactions with the lower aromatic part of the cavitand, and two bifurcated $\mathrm{C}-\mathrm{H} \cdots \mathrm{N}$ interactions with the nitrogen atoms of two adjacent quinoxaline moieties. The shortest distance of a carbon atom of the guest from the mean plane passing through the $\mathrm{O} 1 / \mathrm{O} 2$ groups of atoms is 1.268 (8) А․

\section{Synthesis and crystallization}

All commercial reagents were ACS reagent grade and used as received. Solvents were dried and distilled using standard procedures. ${ }^{1} \mathrm{H}$ NMR spectra were recorded on Bruker Avance 300 (300 MHz) and on Bruker Avance 400 (400 MHz) spectrometers. All chemical shifts $(\delta)$ were reported in parts per million ( $\mathrm{ppm}$ ) relative to proton resonances resulting from incomplete deuteration of NMR solvents. The Matrix-assisted laser desorption/ionization analyses (MALDI TOF-TOF)

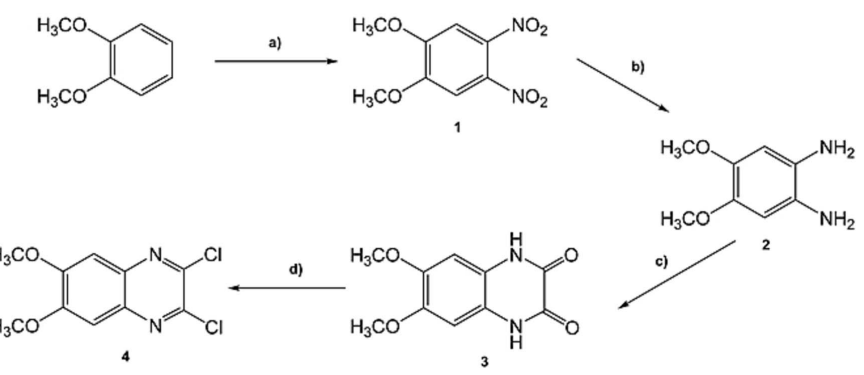

Figure 7

Synthesis of 4: a) $\mathrm{HNO}_{3} 65 \%, 373 \mathrm{~K}, 8 \mathrm{~h}, 80 \%$; b) $\mathrm{Pd} / \mathrm{C} 10 \%, \mathrm{H}_{2} 3$ bar, EtOH, RT, 24 h, 100\%; c) Oxalic acid, $\mathrm{HCl} 4$ N, $373 \mathrm{~K}, 16$ h, 77\%; d) $\mathrm{POCl}_{3}$, dichloroethane, $363 \mathrm{~K}, 16 \mathrm{~h}, 80 \%$. were performed on an AB SCIEX MALDI TOF-TOF 4800 Plus using $\alpha$-cyano-4-hydroxycinnamic acid as a matrix. The GC-Mass analyses were performed on a Hewlett-Packard Agilent 6890 series equipped in Supelcoß SLBTM 5ms column and Hewlett-Packard 5973 MS Selective Mass Detector.

Cavitand QxCav (7) was prepared according to the following convergent synthetic approach: (i) synthesis of the 2,3-dichloro-6,7-dimethoxy quinoxaline bridging unit 4 (Fig. 7); (ii) introduction of the dimethoxy-functionalized quinoxaline bridging unit onto the resorcinarene skeleton, deprotection of the methoxy groups and subsequent ring closure (Fig. 8).

The multistep synthesis of $\mathbf{4}$ started with nitration of veratrole following an electrophilic aromatic substitution reaction in concentrated nitric acid, under reflux. The obtained 1,2dimethoxy-4,5-dinitro benzene (1) was successively reduced using a catalytic amount of metallic Pd on activated carbon in an $\mathrm{H}_{2}$ atmosphere to give 1,2-dimethoxy-4,5-diamino benzene (2). Due to the high reactivity of amino groups, compound 2 was used without any further purification for a condensation with oxalic acid under acidic conditions to give heterocycle 3 . The final step was the chlorination of the 6,7-dimethoxyquinoxaline-2,3-dione (3) in the presence of $\mathrm{POCl}_{3}$ as chlorinating agent, dimethylformamide as catalyst and dichloroethane as solvent. The functionalized bridging unit $\mathbf{4}$ was obtained in $80 \%$ yield after column chromatography.

As regards the resorcinarene scaffold $\left(\mathrm{Res}\left[\mathrm{H}, \mathrm{C}_{6} \mathrm{H}_{13}\right]\right)$ for the preparation of the cavitand receptor, the one with hexyl

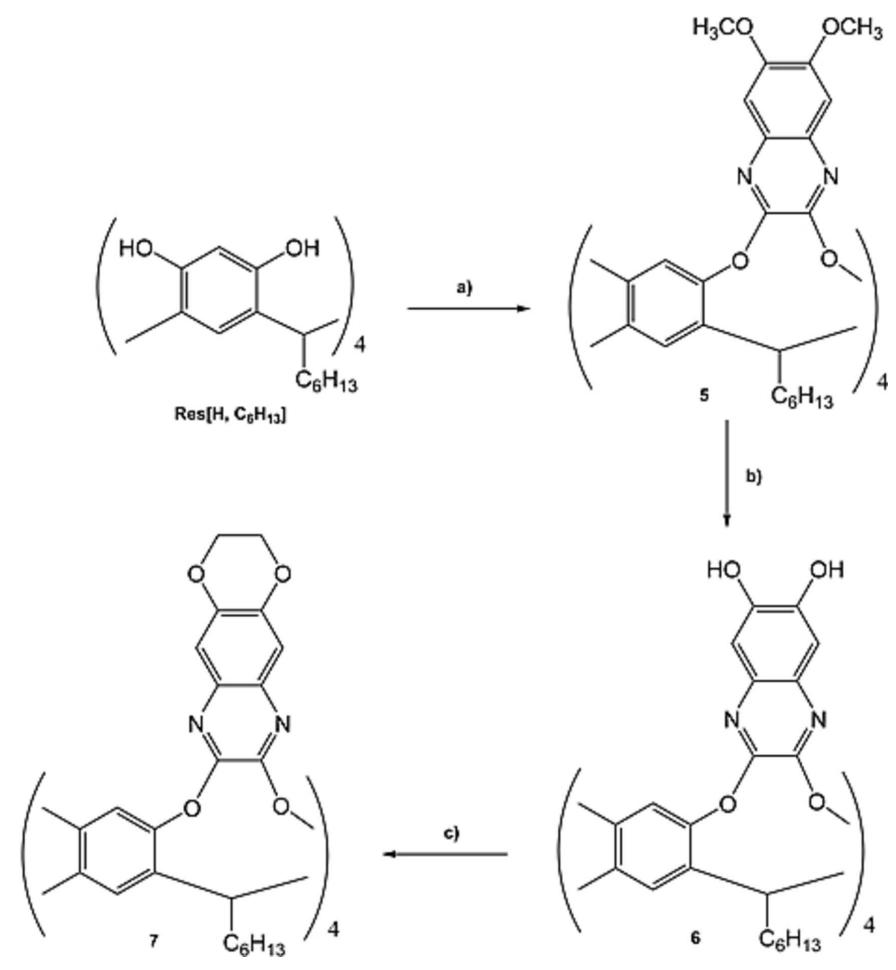

Figure 8

Synthesis of 7: a) 4, $\mathrm{K}_{2} \mathrm{CO}_{3}$, DMF, $393 \mathrm{~K}$ under microwave irradiation $(300 \mathrm{~W}), 2 \mathrm{~h}, 92 \%$; b) $\mathrm{BBr}_{3}$, dry chloroform, $353 \mathrm{~K}, 24 \mathrm{~h}, 100 \%$; c) ethylene glycol ditosylate, $\mathrm{Cs}_{2} \mathrm{CO}_{3}, \mathrm{DMF}, 393 \mathrm{~K}$ under microwave irradiation $(300 \mathrm{~W}), 1.5 \mathrm{~h}, 90 \%$. 
feet was chosen as a compromise between solubility, which helps in the purification of intermediates and final products, and ease of crystallization. The synthesis consists of three steps (Fig. 8): firstly the hexyl-footed resorcinarene $\mathbf{5}$ (Tunstad et al., 1989) was fourfold bridged with the 2,3-dichloro-5,8dimethoxy quinoxaline (4) under microwave irradiation, leading to octamethoxyquinoxaline cavitand (5) in $92 \%$ yield. The ${ }^{1} \mathrm{H}$ NMR studies showed the fluctional vase-kite conformation of the cavitand $\mathbf{5}$, due to the presence of the methoxy groups in the 6,7 positions relative to the quinoxaline moiety. The purified cavitand 6 was successively reacted with a Lewis acid $\left(\mathrm{BBr}_{3}\right)$ in dry chloroform under reflux, to cleave the methyl protecting groups of the quinoxaline walls. The deprotection of eight $\mathrm{CH}_{3}$ groups influences the cavitand conformation, as observed by the ${ }^{1} \mathrm{H}$ NMR analysis, and the octahydroxy cavitand $\mathbf{6}$ is in the pure vase conformation. This change is due to the presence of hydrogen bonding between the hydroxyl groups placed at the cavity entrance. This strong interaction tightens the cavity, holding it in the vase form. The last reaction step was the closure of the 1,4 dioxane ring by reacting the octahydroxy cavitand $\mathbf{6}$ and ethylene glycol ditosylate under microwave irradiation in the presence of $\mathrm{Cs}_{2} \mathrm{CO}_{3}$ as base and dimethylformamide as solvent. Both ${ }^{1} \mathrm{H}$ NMR and MALDI TOF-TOF analyses confirmed the formation of the desired compound.

1,2-Dimethoxy-4,5-dinitro benzene (1): 1,2-Dimethoxy benzene $(40 \mathrm{mmol})$ was added dropwise into a flask containing an aqueous solution of $\mathrm{HNO}_{3} 65 \%(25 \mathrm{~mL})$ and stirred for $1 \mathrm{~h}$ at RT. A yellow precipitate was formed and the reaction was stirred at $373 \mathrm{~K}$ for an additional $8 \mathrm{~h}$. The reaction was cooled to RT and the yellow emulsion was poured into a beaker containing ice-cooled water, filtered and dried under vacuum. The pure product $\mathbf{1}$ was obtained by a threefold recrystallization from glacial acetic acid in $80 \%$ yield. ${ }^{1} \mathrm{H}$ NMR $\left(400 \mathrm{MHz}, \mathrm{CDCl}_{3}\right): \delta=4.05\left(s, 6 \mathrm{H}, \mathbf{C H}_{3} \mathrm{OAr}\right), 7.35(s, 2 \mathrm{H}$, ArH). GC-MS: $m / z 229[M]^{+}$.

1,2-Dimethoxy-4,5-diamino benzene (2): To a suspension of compound $\mathbf{1}(30 \mathrm{mmol})$ in absolute ethanol $(50 \mathrm{~mL})$ a catalytic amount of palladium on charcoal $(10 \%, w / w)$ was added. The reactor was mounted in a PARR hydrogenation apparatus and air atmosphere was replaced with $\mathrm{H}_{2}$ at 3 bar. The reaction was stirred at RT for $24 \mathrm{~h}$. The product was filtered through celite, washed with ethanol and the solvent was removed under reduced pressure obtaining the final product 2 in quantitative yield. ${ }^{1} \mathrm{H}$ NMR $\left(400 \mathrm{MHz}, \mathrm{CDCl}_{3}\right): \delta(\mathrm{ppm})=3.25$ ( $\left.b s, 4 \mathrm{H}, \mathbf{H}_{2} \mathrm{NAr}\right), 3.80\left(s, 6 \mathrm{H}, \mathbf{C H}_{3} \mathrm{OAr}\right), 6.40(s, 2 \mathrm{H}, \operatorname{Ar} \mathbf{H})$. GC-MS: $m / z=169[M]^{+}$.

6,7-Dimethoxyquinoxaline-2,3-dione (3): A solution of compound 2 in $4 N \mathrm{HCl}$ ( $26 \mathrm{mmol}, 1$ eq.) was added to a stirring solution of oxalic acid ( $34 \mathrm{mmol}, 1,3$ eq.) in a $4 \mathrm{~N} \mathrm{HCl}$ solution $(33 \mathrm{~mL})$ and refluxed for $16 \mathrm{~h}$. After cooling to RT, the formed precipitate was filtered and dried under vacuum, giving the desired product 3 in $77 \%$ yield. ${ }^{1} \mathrm{H}-\mathrm{NMR}(300 \mathrm{MHz}$, DMSO- $\left.d_{6}\right): \delta(\mathrm{ppm})=3.65\left(s, 6 \mathrm{H}, \mathbf{C H}_{3} \mathrm{OAr}\right), 6.72(s, 2 \mathrm{H}$, $\operatorname{ArH}), 11.70(s, 2 \mathrm{H}, \mathrm{CNHC})$. GC-MS: $m / z=223[M]^{+}$.

2,3-Dicloro-6,7-dimethoxyquinoxaline (4): 6,7-Dimethoxyquinoxaline-2,3-dione 3 ( $20 \mathrm{mmol}, 1$ eq.), $\mathrm{POCl}_{3}(400 \mathrm{mmol}$,
20 eq.) and three drops of dry DMF were added into dichloroethane $(100 \mathrm{~mL})$ and stirred at $363 \mathrm{~K}$ for $16 \mathrm{~h}$. Subsequently, the solvent was removed under vacuum and the obtained solid was dissolved in dichloromethane and filtered through celite. The crude product was purified by flash chromatography giving the pure compound $\mathbf{4}$ in $80 \%$ yield. ${ }^{1} \mathrm{H}-\mathrm{NMR}\left(400 \mathrm{MHz}, \mathrm{CDCl}_{3}\right): \delta=4.04\left(s, 6 \mathrm{H}, \mathbf{C H}_{3} \mathrm{OAr}\right), 7.25$ $(s, 2 \mathrm{H}, \mathrm{ArH})$. GC-MS: $m / z=260[\mathrm{M}]^{+}$.

Octamethoxy quinoxaline cavitand (5): Resorcinarene $\operatorname{Res}\left[\mathbf{H}, \mathbf{C}_{\mathbf{6}} \mathbf{H}_{\mathbf{1 3}}\right]$ (0.35 mmol, 1eq.), 2,3-dichloro-6,7-dimethoxyquinoxaline 4 ( $0.51 \mathrm{mmol}, 4,5$ eq.), dry $\mathrm{K}_{2} \mathrm{CO}_{3}(1.87 \mathrm{mmol}$, 16 eq.) and dry DMF were added into an oven-dried microwave vessel under an $\mathrm{Ar}$ atmosphere and reacted under microwave irradiation at $393 \mathrm{~K}$ for $2 \mathrm{~h}$. Afterwards, the mixture was extracted with dichloromethane $/ \mathrm{H}_{2} \mathrm{O}$ and the organic fractions were collected, dried over $\mathrm{Na}_{2} \mathrm{SO}_{4}$ and the solvent was removed under reduced pressure. The crude product was purified by flash chromatography affording cavitand 5 in $92 \%$ yield. ${ }^{1} \mathrm{H}-\mathrm{NMR}\left(300 \mathrm{MHz}, \mathrm{CD}_{2} \mathrm{Cl}_{2}\right)$ - fluxional vase conformation: $\delta=0.88(t, 12 \mathrm{H}, \boldsymbol{J}=6.5 \mathrm{~Hz}$, $\left.\mathbf{C H}_{3} \mathrm{CH}_{2} \mathrm{CH}_{2}\right), 1.23-1.32\left(\mathrm{~m}, 32 \mathrm{H},-\mathbf{C H}_{2^{-}}\right), 2.16(b q, 8 \mathrm{H}$, $\left.\mathrm{CHCH}_{2} \mathrm{CH}_{2}\right), 4.04\left(s, 24 \mathrm{H}, \quad \mathbf{C} \mathbf{H}_{3} \mathrm{OAr}\right), 4.48$ (bt, $4 \mathrm{H}$, $\left.\mathbf{C H C H}_{2} \mathrm{CH}_{2}\right), 7.02\left(s, 4 \mathrm{H}, \mathrm{Ar} \mathbf{H}_{\text {down }}\right), 7.24\left(s, 8 \mathrm{H}, \mathbf{C H}_{3} \mathrm{OAr} \mathbf{H}_{2}\right)$ $7.48\left(s, 4 \mathrm{H}, \operatorname{Ar} \mathbf{H}_{\text {up }}\right)$. MALDI TOF-TOF: $m / z=1569[M]^{+}$.

Octahydroxy quinoxaline cavitand (6): Cavitand 5 $(0.03 \mathrm{mmol}, 1$ eq.) was dissolved in dry chloroform $(10 \mathrm{~mL})$ and $\mathrm{BBr}_{3}$ (3.80 mmol, 120 eq.) was added dropwise under an $\mathrm{Ar}$ atmosphere. The mixture was stirred at $353 \mathrm{~K}$ for $24 \mathrm{~h}$ and $\mathrm{H}_{2} \mathrm{O}(30 \mathrm{~mL})$ was added into a boiling solution. After cooling down to room temperature, chloroform was removed and the yellow solid was sonicated with $1 \mathrm{~N} \mathrm{HCl}$, filtrated and dried under vacuum obtaining the final product 6 in quantitative yield. ${ }^{1} \mathrm{H}-\mathrm{NMR}\left(300 \mathrm{MHz}\right.$, DMSO- $\left.d_{6}\right)$ - vase conformation: $\delta=0.85\left(t, 12 \mathrm{H}, \boldsymbol{J}=6.3 \mathrm{~Hz}, \mathbf{C H}_{\mathbf{3}} \mathrm{CH}_{2} \mathrm{CH}_{2}\right), 1.12-1.37(m, 32 \mathrm{H}$, $\left.{ }_{-} \mathbf{C H}_{2}-\right), 2.37\left(b q, 8 \mathrm{H} \mathrm{CHCH} \mathbf{C H}_{2}\right), 5.38\left(b t, 4 \mathrm{H}, \mathbf{C H C H}_{2} \mathrm{CH}_{2}\right)$, $7.08\left(s, 8 \mathrm{H}, \mathbf{C H}_{3} \mathrm{OArH}_{2}\right), 7.69\left(s, 4 \mathrm{H}, \operatorname{ArH}_{\text {down }}\right), 7.84(s, 4 \mathrm{H}$, $\left.\operatorname{ArH}_{\text {up }}\right), 9.94(s, 8 \mathrm{H}$, ArOH). MALDI TOF-TOF: $m / z=1457$ $[M]^{+}$.

DeepQxCav (7): Cavitand 6 ( $0.052 \mathrm{mmol}, 1$ eq. $)$, ethylene glycol ditosylate $\left(0.52 \mathrm{mmol}, 10\right.$ eq.), dry $\mathrm{Cs}_{2} \mathrm{CO}_{3}(0.63 \mathrm{mmol}$, 12 eq.) and dry DMF (5 mL) were added into an oven-dried microwave vessel under an Ar atmosphere and reacted under microwave irradiation at $393 \mathrm{~K}$ for $1.5 \mathrm{~h}$. The reaction was quenched in water and extracted with $\mathrm{DCM} / \mathrm{H}_{2} \mathrm{O}$. The organic fractions were collected and dried over $\mathrm{Na}_{2} \mathrm{SO}_{4}$. After filtration the solvent was removed under reduced pressure and the crude was purified by flash chromatography. The final product $\mathbf{7}$ was obtained in $90 \%$ yield. ${ }^{\mathbf{1}} \mathbf{H}-\mathbf{N M R}\left(300 \mathrm{MHz}\right.$, DMSO- $d_{6}$ ) - vase conformation: $\delta=0.86\left(s, 12 \mathrm{H}, \mathbf{C H}_{3} \mathrm{CH}_{2} \mathrm{CH}_{2}\right), 1.20-1.48$ $\left(\mathrm{m}, 32 \mathrm{H},-\mathbf{C H}_{\mathbf{2}^{-}}\right), 2.40\left(\mathrm{bq}, 8 \mathrm{H} \mathbf{C H} \mathbf{C H}_{\mathbf{2}} \mathrm{CH}_{2}\right), 4.28-4.42(\mathrm{~m}$, $\left.16 \mathrm{H}, \mathrm{ArOCH}_{\mathbf{2}} \mathbf{C H}_{\mathbf{2}} \mathrm{O}\right), 5.48\left(t, 4 \mathrm{H}, \boldsymbol{J}=7.6 \mathrm{~Hz}, \mathbf{C H C H}_{2} \mathrm{CH}_{2}\right)$, $7.20\left(s, 8 \mathrm{H}, \operatorname{ArH}_{2}\right), 7.74\left(s, 4 \mathrm{H}, \operatorname{Ar} \mathbf{H}_{\text {down }}\right), 7.89\left(s, 4 \mathrm{H}, \operatorname{Ar} \mathbf{H}_{\text {up }}\right)$. MALDI TOF-TOF: calculated for $\mathrm{C}_{92} \mathrm{H}_{88} \mathrm{~N}_{8} \mathrm{O}_{16}[M]^{+} \mathrm{m} / \mathrm{z}=$ 1560.6318; found $\mathrm{m} / z=1560.8065$.

Prismatic, colourless single crystals of the title compound suitable for X-ray analysis were obtained by slow evaporation of a benzene solution. 
Table 3

Experimental details.

\begin{tabular}{|c|c|}
\hline \multicolumn{2}{|l|}{ Crystal data } \\
\hline Chemical formula & $\mathrm{C}_{92} \mathrm{H}_{88} \mathrm{O}_{16} \mathrm{~N}_{8} \cdot \mathrm{C}_{6} \mathrm{H}_{6}$ \\
\hline$M_{\mathrm{r}}$ & 1639.81 \\
\hline Crystal system, space group & Monoclinic, $C 2 / c$ \\
\hline Temperature $(\mathrm{K})$ & 190 \\
\hline$a, b, c(\AA)$ & 19.173 (1), 20.756 (1), 21.771 (2) \\
\hline$\beta\left(^{\circ}\right)$ & $110.718(2)$ \\
\hline$V\left(\AA^{3}\right)$ & $8103.6(9)$ \\
\hline$Z$ & 4 \\
\hline Radiation type & Мо $K \alpha$ \\
\hline$\mu\left(\mathrm{mm}^{-1}\right)$ & 0.09 \\
\hline Crystal size $(\mathrm{mm})$ & $0.13 \times 0.10 \times 0.08$ \\
\hline \multicolumn{2}{|l|}{ Data collection } \\
\hline Diffractometer & Bruker APEXII CCD \\
\hline Absorption correction & $\begin{array}{l}\text { Multi-scan (TWINABS; Sheldrick, } \\
\text { 2008) }\end{array}$ \\
\hline$T_{\min }, T_{\max }$ & $0.671,0.746$ \\
\hline $\begin{array}{l}\text { No. of measured, independent and } \\
\text { observed }[I>2 \sigma(I)] \text { reflections }\end{array}$ & $8326,8326,6387$ \\
\hline$R_{\text {int }}$ & 0.0 \\
\hline$(\sin \theta / \lambda)_{\max }\left(\AA^{-1}\right)$ & 0.627 \\
\hline \multicolumn{2}{|l|}{ Refinement } \\
\hline$R\left[F^{2}>2 \sigma\left(F^{2}\right)\right], w R\left(F^{2}\right), S$ & $0.046,0.119,1.02$ \\
\hline No. of reflections & 8326 \\
\hline No. of parameters & 573 \\
\hline No. of restraints & 22 \\
\hline $\mathrm{H}$-atom treatment & $\mathrm{H}$-atom parameters constrained \\
\hline$\Delta \rho_{\max }, \Delta \rho_{\min }\left(\mathrm{e} \AA^{-3}\right)$ & $0.35,-0.26$ \\
\hline
\end{tabular}

Computer programs: APEX2 and SAINT (Bruker, 2008), SIR97 (Altomare et al., 1999), SHELXL2014 (Sheldrick, 2015), Mercury (Macrae et al., 2006), WinGX (Farrugia, 2012), PARST (Nardelli, 1995) and publCIF (Westrip, 2010).

\section{Refinement}

Crystal data, data collection and structure refinement details are summarized in Table 3 .

The structure of the title compound was refined as a twocomponent twin with a BASF parameter of 0.572 (1). The last cycle of refinement was performed with a HKLF 5 dataset containing 12410 corrected reflections constructed from all observations involving domain 2 .

A carbon atom $(\mathrm{C} 23 B)$ of one of the upper 1,4 dioxane rings was found to be disordered over two positions with occupancies of 0.547 (17) and 0.453 (17). The benzene guest was found disordered over two equally populated positions. For one of the two orientations (atoms $\mathrm{C} 1 S, \mathrm{C} 2 S, \mathrm{C} 3 S$ and their symmetry-generated analogues), the aromatic ring was modelled by fixing the bond distances to 1.380 (1) $\AA$. The SIMU restraint (Sheldrick, 2015) was applied to atoms C4S$\mathrm{C} 7 \mathrm{~S}$ of the second orientation.

The carbon-bound $\mathrm{H}$ atoms were placed in calculated positions and refined isotropically using a riding model with $\mathrm{C}-\mathrm{H}$ ranging from 0.95 to $0.99 \AA$ and $U_{\text {iso }}(\mathrm{H})$ set to $1.2-$ $1.5 U_{\mathrm{eq}}(\mathrm{C})$.

\section{Acknowledgements}

The Centro Interfacoltà di Misure 'G. Casnati' and the 'Laboratorio di Strutturistica Mario Nardelli' of the Univer- sity of Parma are kindly acknowledged for the use of the NMR and MALDI-MS facilities, and of the diffractometer.

\section{References}

Altomare, A., Burla, M. C., Camalli, M., Cascarano, G. L., Giacovazzo, C., Guagliardi, A., Moliterni, A. G. G., Polidori, G. \& Spagna, R. (1999). J. Appl. Cryst. 32, 115-119.

Azov, V. A., Skinner, P. J., Yamakoshi, Y., Seiler, P., Gramlich, V. \& Diederich, F. (2003). Helv. Chim. Acta, 86, 3648-3670.

Ballistreri, F. P., Brancatelli, G., Demitri, N., Geremia, S., Guldi, D. M., Melchionna, M., Pappalardo, A., Prato, M., Tomaselli, G. A. \& Sfrazzetto, G. T. (2016). Supramol. Chem. 28, 601-607.

Bertani, F., Riboni, N., Bianchi, F., Brancatelli, G., Sterner, E. S., Pinalli, R., Geremia, S., Swager, T. M. \& Dalcanale, E. (2016). Chem. Eur. J. 22, 3312-3319.

Brekalo, I., Kane, C. M., Ley, A. N., Ramirez, J. R., Friščić, T. \& Holman, K. T. (2018). J. Am. Chem. Soc. 140, 10104-10108.

Bruker (2008). APEX2, SAINT and SADABS. Bruker AXS Inc., Madison, Wisconsin, USA.

Cram, D. J. (1983). Science, 219, 1177-1183.

Cram, D. J. \& Cram, J. M. (1994). Container Molecules and their Guests, Monographs in Supramolecular Chemistry, edited by J. F. Stoddart, Vol. 4. Cambridge: Royal Society of Chemistry.

Farrugia, L. J. (2012). J. Appl. Cryst. 45, 849-854.

Groom, C. R., Bruno, I. J., Lightfoot, M. P. \& Ward, S. C. (2016). Acta Cryst. B72, 171-179.

Kane, C. M., Ugono, O., Barbour, L. J. \& Holman, K. T. (2015). Chem. Mater. 27, 7337-7354.

Lee, J., Perez, L., Liu, Y., Wang, H., Hooley, R. J. \& Zhong, W. (2018). Anal. Chem. 90, 1881-1888.

Liu, Y., Lee, J., Perez, L., Gill, A. D., Hooley, R. J. \& Zhong, W. (2018). J. Am. Chem. Soc. 140, 13869-13877.

Macrae, C. F., Edgington, P. R., McCabe, P., Pidcock, E., Shields, G. P., Taylor, R., Towler, M. \& van de Streek, J. (2006). J. Appl. Cryst. 39, 453-457.

Melegari, M., Massera, C., Pinalli, R., Yebeutchou, R. M. \& Dalcanale, E. (2013). Sens. Actuators B Chem. 179, 74-80.

Nardelli, M. (1995). J. Appl. Cryst. 28, 659.

Pinalli, R., Barboza, T., Bianchi, F., Massera, C., Ugozzoli, F. \& Dalcanale, E. (2013). Supramol. Chem. 25, 682-687.

Pinalli, R., Dalcanale, E., Ugozzoli, F. \& Massera, C. (2016). CrystEngComm, 18, 5788-5802.

Pinalli, R., Pedrini, A. \& Dalcanale, E. (2018). Chem. Eur. J. 24, 10101019.

Sheldrick, G. M. (2008). TWINABS. University of Göttingen, Germany.

Sheldrick, G. M. (2015). Acta Cryst. C71, 3-8.

Soncini, P., Bonsignore, S., Dalcanale, E. \& Ugozzoli, F. (1992). J. Org. Chem. 57, 4608-4612.

Trzciński, J. W., Pinalli, R., Riboni, N., Pedrini, A., Bianchi, F., Zampolli, S., Elmi, I., Massera, C., Ugozzoli, F. \& Dalcanale, E. (2017). ACS Sensors 2, 590-598.

Tudisco, C., Fragalà, M. E., Giuffrida, A. E., Bertani, F., Pinalli, R., Dalcanale, E., Compagnini, G. \& Condorelli, G. G. (2016). J. Phys. Chem. C, 120, 12611-12617.

Tunstad, L., Tucker, J. A., Dalcanale, E., Weiser, J., Bryant, J. A., Sherman, J. C., Helgeson, R. C., Knobler, C. B. \& Cram, D. J. (1989). J. Org. Chem. 54, 1305-1312.

Wagner, G., Arion, V. B., Brecker, L., Krantz, C., Mieusset, J.-L. \& Brinker, U. H. (2009). Org. Lett. 11, 3056-3058.

Westrip, S. P. (2010). J. Appl. Cryst. 43, 920-925. 


\section{supporting information}

Acta Cryst. (2019). E75, 103-108 [https://doi.org/10.1107/S2056989018017784]

\section{A new, deep quinoxaline-based cavitand receptor for the complexation of benzene}

\section{Roberta Pinalli, Jakub W. Trzciński, Enrico Dalcanale and Chiara Massera}

Computing details

Data collection: APEX2 (Bruker, 2008); cell refinement: APEX2 (Bruker, 2008); data reduction: SAINT (Bruker, 2008) and SADABS (Bruker, 2008); program(s) used to solve structure: SIR97 (Altomare et al., 1999); program(s) used to refine structure: SHELXL2014 (Sheldrick, 2015); molecular graphics: Mercury (Macrae et al., 2006); software used to prepare material for publication: WinGX (Farrugia, 2012), PARST (Nardelli, 1995) and publCIF (Westrip, 2010).

2,8,14,20-Tetrahexyl-4,24:6,10:12,16:18,22-O,O'-tetrakis[2,3-dihydro-[1,4]dioxino[2,3-g]quinoxalin-7,8diyl]resorcin[4]arene benzene monosolvate

\section{Crystal data}

$\mathrm{C}_{92} \mathrm{H}_{88} \mathrm{O}_{16} \mathrm{~N}_{8} \cdot \mathrm{C}_{6} \mathrm{H}_{6}$

$M_{r}=1639.81$

Monoclinic, $C 2 / c$

$a=19.173(1) \AA$

$b=20.756(1) \AA$

$c=21.771(2) \AA$

$\beta=110.718(2)^{\circ}$

$V=8103.6(9) \AA^{3}$

$Z=4$

\section{Data collection}

Bruker APEXII CCD

diffractometer

Radiation source: fine-focus sealed tube

Graphite monochromator

$\omega$ scan

Absorption correction: multi-scan

(TWINABS; Sheldrick, 2008)

$T_{\min }=0.671, T_{\max }=0.746$

\section{Refinement}

Refinement on $F^{2}$

Least-squares matrix: full

$R\left[F^{2}>2 \sigma\left(F^{2}\right)\right]=0.046$

$w R\left(F^{2}\right)=0.119$

$S=1.02$

8326 reflections

573 parameters

22 restraints
$F(000)=3464$

$D_{\mathrm{x}}=1.344 \mathrm{Mg} \mathrm{m}^{-3}$

Mo $K \alpha$ radiation, $\lambda=0.71073 \AA$

Cell parameters from 1365 reflections

$\theta=1-26.5^{\circ}$

$\mu=0.09 \mathrm{~mm}^{-1}$

$T=190 \mathrm{~K}$

Prismatic, colourless

$0.13 \times 0.10 \times 0.08 \mathrm{~mm}$

8326 measured reflections

8326 independent reflections

6387 reflections with $I>2 \sigma(I)$

$R_{\text {int }}=0.0$

$\theta_{\max }=26.5^{\circ}, \theta_{\min }=1.5^{\circ}$

$h=-24 \rightarrow 22$

$k=0 \rightarrow 25$

$l=0 \rightarrow 27$

Primary atom site location: structure-invariant direct methods

Hydrogen site location: inferred from neighbouring sites

$\mathrm{H}$-atom parameters constrained

$w=1 /\left[\sigma^{2}\left(F_{\mathrm{o}}^{2}\right)+(0.0582 P)^{2}+3.3175 P\right]$

where $P=\left(F_{\mathrm{o}}{ }^{2}+2 F_{\mathrm{c}}{ }^{2}\right) / 3$

$(\Delta / \sigma)_{\max }=0.001$ 
$\Delta \rho_{\max }=0.35$ e $\AA^{-3}$

$\Delta \rho_{\min }=-0.26$ e $\AA^{-3}$

Special details

Geometry. All esds (except the esd in the dihedral angle between two 1.s. planes) are estimated using the full covariance matrix. The cell esds are taken into account individually in the estimation of esds in distances, angles and torsion angles; correlations between esds in cell parameters are only used when they are defined by crystal symmetry. An approximate (isotropic) treatment of cell esds is used for estimating esds involving l.s. planes.

Refinement. Refined as a 2-component twin. 8600 Corrected reflections written to file twin4.hkl Reflections merged according to point-group 2/m Minimum and maximum apparent transmission: 0.6714790 .745373 Additional spherical absorption correction applied with $m u^{*} \mathrm{r}=0.2000$

HKLF 5 dataset constructed from all observations involving domain 212410 Corrected reflections written to file twin5.hkl Reflections merged according to point-group $2 / \mathrm{m}$ Single reflections that also occur in composites omitted Minimum and maximum apparent transmission: 0.6712670 .745373 Additional spherical absorption correction applied with $m u^{*} r=0.200$

Fractional atomic coordinates and isotropic or equivalent isotropic displacement parameters $\left(\AA^{2}\right)$

\begin{tabular}{|c|c|c|c|c|c|}
\hline & $x$ & $y$ & $z$ & $U_{\text {iso }} * / U_{\text {eq }}$ & Occ. $(<1)$ \\
\hline $\mathrm{C} 1 \mathrm{~S}$ & $0.4811(3)$ & $0.2252(2)$ & $0.2717(2)$ & $0.0445(14)$ & 0.5 \\
\hline $\mathrm{H} 1 \mathrm{~S}$ & 0.4682 & 0.2647 & 0.2870 & $0.053^{*}$ & 0.5 \\
\hline $\mathrm{C} 3 \mathrm{~S}$ & $0.4808(3)$ & $0.1094(2)$ & $0.2715(2)$ & $0.055(2)$ & 0.5 \\
\hline $\mathrm{H} 3 \mathrm{~S}$ & 0.4673 & 0.0699 & 0.2863 & $0.066^{*}$ & 0.5 \\
\hline $\mathrm{C} 2 \mathrm{~S}$ & 0.4622 (4) & 0.16731 (19) & 0.2928 (4) & $0.070(3)$ & 0.5 \\
\hline $\mathrm{H} 2 \mathrm{~S}$ & 0.4359 & 0.1673 & 0.3225 & $0.084 *$ & 0.5 \\
\hline $\mathrm{C} 4 \mathrm{~S}$ & 0.5000 & $0.0994(8)$ & 0.2500 & $0.070(2)$ & 0.5 \\
\hline $\mathrm{H} 4 \mathrm{~S}$ & 0.5000 & 0.0536 & 0.2500 & $0.083^{*}$ & 0.5 \\
\hline C7S & 0.5000 & $0.2312(6)$ & 0.2500 & $0.070(2)$ & 0.5 \\
\hline $\mathrm{H} 7 \mathrm{~S}$ & 0.5000 & 0.2769 & 0.2500 & $0.083^{*}$ & 0.5 \\
\hline $\mathrm{C} 5 \mathrm{~S}$ & $0.4655(7)$ & $0.1331(4)$ & $0.2850(6)$ & $0.070(2)$ & 0.5 \\
\hline $\mathrm{H} 5 \mathrm{~S}$ & 0.4407 & 0.1107 & 0.3093 & $0.083^{*}$ & 0.5 \\
\hline $\mathrm{C} 6 \mathrm{~S}$ & $0.4664(5)$ & 0.1979 (4) & $0.2852(5)$ & $0.070(2)$ & 0.5 \\
\hline $\mathrm{H} 6 \mathrm{~S}$ & 0.4430 & 0.2207 & 0.3105 & $0.083 *$ & 0.5 \\
\hline N1A & $0.59064(9)$ & $0.81836(7)$ & $0.54916(8)$ & $0.0263(4)$ & \\
\hline $\mathrm{N} 2 \mathrm{~A}$ & $0.71761(9)$ & $0.81762(8)$ & $0.66567(8)$ & $0.0281(4)$ & \\
\hline O1A & $0.59123(7)$ & $0.70686(6)$ & $0.55285(6)$ & $0.0256(3)$ & \\
\hline $\mathrm{O} 2 \mathrm{~A}$ & $0.71490(7)$ & $0.70662(6)$ & $0.66212(6)$ & $0.0271(3)$ & \\
\hline O3A & $0.57670(9)$ & $1.04593(6)$ & $0.56278(7)$ & 0.0364 (4) & \\
\hline $\mathrm{O} 4 \mathrm{~A}$ & $0.69554(9)$ & $1.04353(7)$ & $0.68594(7)$ & $0.0382(4)$ & \\
\hline C1A & $0.47418(11)$ & $0.61563(8)$ & $0.62389(8)$ & $0.0213(4)$ & \\
\hline H1A & 0.4779 & 0.5795 & 0.6517 & $0.026^{*}$ & \\
\hline $\mathrm{C} 2 \mathrm{~A}$ & $0.53807(10)$ & $0.63498(8)$ & $0.61211(9)$ & $0.0216(4)$ & \\
\hline C3A & $0.52994(11)$ & $0.68768(9)$ & $0.57033(9)$ & 0.0219 (4) & \\
\hline $\mathrm{C} 4 \mathrm{~A}$ & $0.46352(11)$ & $0.72085(8)$ & $0.54400(9)$ & 0.0217 (4) & \\
\hline $\mathrm{H} 4 \mathrm{~A}$ & 0.4598 & 0.7571 & 0.5164 & $0.026^{*}$ & \\
\hline C5A & $0.40255(10)$ & $0.70036(8)$ & $0.55851(9)$ & 0.0214 (4) & \\
\hline C6A & $0.40516(11)$ & $0.64632(8)$ & $0.59717(9)$ & $0.0206(4)$ & \\
\hline C7A & $0.61328(10)$ & $0.60203(9)$ & $0.64580(9)$ & $0.0222(4)$ & \\
\hline H7A & 0.6444 & 0.6108 & 0.6183 & $0.027^{*}$ & \\
\hline C8A & $0.60921(11)$ & $0.52876(9)$ & $0.65182(9)$ & $0.0259(4)$ & \\
\hline
\end{tabular}




\begin{tabular}{|c|c|c|c|c|}
\hline H8A1 & 0.6605 & 0.5116 & 0.6719 & $0.031 *$ \\
\hline H8A2 & 0.5820 & 0.5186 & 0.6817 & $0.031^{*}$ \\
\hline C9A & $0.57066(13)$ & $0.49489(10)$ & $0.58614(10)$ & $0.0333(5)$ \\
\hline H9A1 & 0.5875 & 0.5149 & 0.5525 & $0.040^{*}$ \\
\hline H9A2 & 0.5162 & 0.5019 & 0.5725 & $0.040 *$ \\
\hline C10A & $0.58578(13)$ & $0.42232(10)$ & $0.58786(10)$ & $0.0352(5)$ \\
\hline H10A & 0.5548 & 0.4037 & 0.5450 & $0.042 *$ \\
\hline H10B & 0.6387 & 0.4157 & 0.5930 & $0.042 *$ \\
\hline C11A & $0.57026(14)$ & $0.38546(10)$ & $0.64198(11)$ & $0.0375(5)$ \\
\hline H11A & 0.5193 & 0.3960 & 0.6405 & $0.045^{*}$ \\
\hline H11B & 0.6058 & 0.3998 & 0.6851 & $0.045^{*}$ \\
\hline $\mathrm{C} 12 \mathrm{~A}$ & $0.57693(14)$ & $0.31275(11)$ & $0.63640(12)$ & $0.0432(6)$ \\
\hline $\mathrm{H} 12 \mathrm{~A}$ & 0.5380 & 0.2978 & 0.5953 & $0.052 *$ \\
\hline H12B & 0.6261 & 0.3026 & 0.6335 & $0.052 *$ \\
\hline C13A & $0.56887(17)$ & $0.27643(12)$ & $0.69372(14)$ & $0.0585(7)$ \\
\hline H13A & 0.5736 & 0.2301 & 0.6875 & $0.088^{*}$ \\
\hline H13B & 0.6079 & 0.2902 & 0.7345 & $0.088 *$ \\
\hline $\mathrm{H} 13 \mathrm{C}$ & 0.5198 & 0.2854 & 0.6963 & $0.088 *$ \\
\hline C14A & $0.62121(11)$ & $0.76567(9)$ & $0.57833(9)$ & $0.0239(4)$ \\
\hline C15A & $0.68585(11)$ & $0.76556(9)$ & 0.63649 (9) & $0.0251(4)$ \\
\hline C16A & $0.68381(11)$ & $0.87427(9)$ & $0.63890(9)$ & $0.0267(4)$ \\
\hline C17A & $0.62093(11)$ & $0.87498(9)$ & $0.57991(9)$ & $0.0261(4)$ \\
\hline C18A & $0.58733(11)$ & $0.93389(9)$ & $0.55450(10)$ & $0.0296(5)$ \\
\hline H18A & 0.5471 & 0.9351 & 0.5136 & $0.036^{*}$ \\
\hline C19A & $0.61237(11)$ & $0.98965(10)$ & $0.58853(10)$ & $0.0289(4)$ \\
\hline $\mathrm{C} 20 \mathrm{~A}$ & $0.67261(11)$ & $0.98849(10)$ & $0.64967(10)$ & $0.0288(5)$ \\
\hline $\mathrm{C} 21 \mathrm{~A}$ & $0.70941(11)$ & $0.93230(10)$ & $0.67279(10)$ & $0.0307(5)$ \\
\hline $\mathrm{H} 21 \mathrm{~A}$ & 0.7523 & 0.9323 & 0.7118 & $0.037^{*}$ \\
\hline $\mathrm{C} 22 \mathrm{~A}$ & $0.61161(14)$ & $1.10373(10)$ & $0.59622(11)$ & $0.0406(6)$ \\
\hline $\mathrm{H} 22 \mathrm{~A}$ & 0.5751 & 1.1395 & 0.5848 & $0.049 *$ \\
\hline $\mathrm{H} 22 \mathrm{~B}$ & 0.6536 & 1.1158 & 0.5821 & $0.049 *$ \\
\hline $\mathrm{C} 23 \mathrm{~A}$ & $0.63970(14)$ & $1.09286(10)$ & $0.66863(12)$ & 0.0407 (6) \\
\hline $\mathrm{H} 23 \mathrm{~A}$ & 0.6611 & 1.1334 & 0.6916 & $0.049 *$ \\
\hline H23B & 0.5979 & 1.0799 & 0.6826 & $0.049^{*}$ \\
\hline N1B & $0.27919(9)$ & $0.84255(8)$ & $0.63477(8)$ & 0.0269 (4) \\
\hline N2B & $0.36596(9)$ & $0.84062(7)$ & $0.55356(8)$ & 0.0268 (4) \\
\hline O1B & $0.25211(7)$ & $0.73485(6)$ & $0.61013(6)$ & $0.0244(3)$ \\
\hline $\mathrm{O} 2 \mathrm{~B}$ & $0.33462(7)$ & $0.73349(6)$ & $0.53150(6)$ & $0.0238(3)$ \\
\hline $\mathrm{O} 3 \mathrm{~B}$ & $0.33383(10)$ & $1.06529(7)$ & $0.67816(8)$ & $0.0456(4)$ \\
\hline O4B & $0.42759(10)$ & $1.06135(7)$ & $0.60250(9)$ & $0.0456(4)$ \\
\hline C1B & $0.64056(10)$ & $0.61566(9)$ & $0.76929(9)$ & $0.0215(4)$ \\
\hline H1B & 0.6100 & 0.5791 & 0.7672 & $0.026^{*}$ \\
\hline $\mathrm{C} 2 \mathrm{~B}$ & $0.32793(10)$ & $0.64723(8)$ & $0.67067(9)$ & $0.0203(4)$ \\
\hline C3B & $0.28589(10)$ & $0.70178(9)$ & $0.66982(9)$ & $0.0217(4)$ \\
\hline C4B & $0.72827(10)$ & $0.72273(9)$ & $0.77536(9)$ & $0.0231(4)$ \\
\hline H4B & 0.7581 & 0.7597 & 0.7773 & $0.028^{*}$ \\
\hline C5B & $0.69772(10)$ & $0.68829(9)$ & $0.71774(9)$ & $0.0223(4)$ \\
\hline C6B & $0.65171(10)$ & $0.63515(8)$ & $0.71207(9)$ & 0.0209 (4) \\
\hline
\end{tabular}




\begin{tabular}{|c|c|c|c|c|c|}
\hline C7B & $0.33514(10)$ & $0.62166(8)$ & $0.60727(9)$ & $0.0217(4)$ & \\
\hline H7B & 0.2921 & 0.6402 & 0.5706 & $0.026^{*}$ & \\
\hline $\mathrm{C} 8 \mathrm{~B}$ & $0.32661(11)$ & $0.54817(8)$ & $0.60047(9)$ & $0.0242(4)$ & \\
\hline H8B1 & 0.3699 & 0.5274 & 0.6340 & $0.029 *$ & \\
\hline H8B2 & 0.2812 & 0.5349 & 0.6088 & $0.029^{*}$ & \\
\hline C9B & $0.32125(13)$ & $0.52490(9)$ & $0.53253(10)$ & $0.0305(5)$ & \\
\hline H9B1 & 0.3715 & 0.5264 & 0.5295 & $0.037^{*}$ & \\
\hline H9B2 & 0.2889 & 0.5549 & 0.4992 & $0.037^{*}$ & \\
\hline $\mathrm{C} 10 \mathrm{~B}$ & $0.29031(13)$ & $0.45675(9)$ & $0.51620(10)$ & $0.0334(5)$ & \\
\hline $\mathrm{H} 10 \mathrm{C}$ & 0.2403 & 0.4554 & 0.5198 & $0.040^{*}$ & \\
\hline H10D & 0.2839 & 0.4475 & 0.4699 & $0.040 *$ & \\
\hline C11B & $0.33720(13)$ & $0.40362(10)$ & 0.55869 (11) & $0.0360(5)$ & \\
\hline $\mathrm{H} 11 \mathrm{C}$ & 0.3424 & 0.4115 & 0.6050 & $0.043^{*}$ & \\
\hline H11D & 0.3877 & 0.4048 & 0.5560 & $0.043 *$ & \\
\hline $\mathrm{C} 12 \mathrm{~B}$ & 0.30369 (13) & $0.33713(9)$ & $0.53828(11)$ & $0.0360(5)$ & \\
\hline $\mathrm{H} 12 \mathrm{C}$ & 0.2527 & 0.3364 & 0.5398 & $0.043^{*}$ & \\
\hline H12D & 0.2995 & 0.3291 & 0.4923 & $0.043^{*}$ & \\
\hline C13B & $0.34868(16)$ & $0.28340(11)$ & $0.58112(14)$ & $0.0519(7)$ & \\
\hline H13D & 0.3242 & 0.2420 & 0.5655 & $0.078 *$ & \\
\hline H13E & 0.3989 & 0.2830 & 0.5791 & $0.078^{*}$ & \\
\hline $\mathrm{H} 13 \mathrm{~F}$ & 0.3520 & 0.2904 & 0.6266 & $0.078^{*}$ & \\
\hline C14B & $0.28771(10)$ & $0.79144(9)$ & $0.60426(9)$ & $0.0232(4)$ & \\
\hline C15B & $0.33131(11)$ & $0.79054(9)$ & $0.56320(9)$ & $0.0231(4)$ & \\
\hline C16B & 0.35949 (11) & $0.89601(9)$ & $0.58592(9)$ & $0.0268(4)$ & \\
\hline C17B & $0.31538(11)$ & $0.89708(9)$ & $0.62631(9)$ & $0.0259(4)$ & \\
\hline C18B & 0.30869 (13) & $0.95474(9)$ & $0.65696(10)$ & $0.0325(5)$ & \\
\hline H18B & 0.2790 & 0.9560 & 0.6839 & $0.039 *$ & \\
\hline C19B & $0.34410(12)$ & $1.00920(9)$ & $0.64875(10)$ & $0.0305(5)$ & \\
\hline C20B & $0.38977(12)$ & $1.00780(9)$ & $0.60987(10)$ & $0.0314(5)$ & \\
\hline $\mathrm{C} 21 \mathrm{~B}$ & $0.39683(13)$ & $0.95212(9)$ & $0.57931(11)$ & $0.0336(5)$ & \\
\hline $\mathrm{H} 21 \mathrm{~B}$ & 0.4274 & 0.9514 & 0.5532 & $0.040 *$ & \\
\hline $\mathrm{C} 22 \mathrm{~B}$ & $0.3660(2)$ & $1.11976(12)$ & $0.66256(19)$ & $0.0798(11)$ & $0.547(17)$ \\
\hline $\mathrm{H} 22 \mathrm{C}$ & 0.3267 & 1.1423 & 0.6267 & $0.096^{*}$ & $0.547(17)$ \\
\hline $\mathrm{H} 22 \mathrm{D}$ & 0.3803 & 1.1486 & 0.7013 & $0.096^{*}$ & $0.547(17)$ \\
\hline C23B & $0.4260(5)$ & $1.1140(3)$ & $0.6441(6)$ & $0.048(2)$ & $0.547(17)$ \\
\hline $\mathrm{H} 23 \mathrm{C}$ & 0.4708 & 1.1108 & 0.6845 & $0.058^{*}$ & $0.547(17)$ \\
\hline $\mathrm{H} 23 \mathrm{D}$ & 0.4305 & 1.1543 & 0.6215 & $0.058^{*}$ & $0.547(17)$ \\
\hline $\mathrm{C} 22 \mathrm{C}$ & $0.3660(2)$ & $1.11976(12)$ & $0.66256(19)$ & $0.0798(11)$ & $0.453(17)$ \\
\hline $\mathrm{H} 22 \mathrm{E}$ & 0.4070 & 1.1330 & 0.7030 & $0.096^{*}$ & $0.453(17)$ \\
\hline $\mathrm{H} 22 \mathrm{~F}$ & 0.3280 & 1.1544 & 0.6525 & $0.096^{*}$ & $0.453(17)$ \\
\hline $\mathrm{C} 23 \mathrm{C}$ & $0.3952(7)$ & $1.1197(3)$ & $0.6112(6)$ & $0.040(2)$ & $0.453(17)$ \\
\hline $\mathrm{H} 23 \mathrm{E}$ & 0.3545 & 1.1306 & 0.5696 & $0.048^{*}$ & $0.453(17)$ \\
\hline $\mathrm{H} 23 \mathrm{~F}$ & 0.4333 & 1.1541 & 0.6200 & $0.048^{*}$ & $0.453(17)$ \\
\hline
\end{tabular}

Atomic displacement parameters $\left(\AA^{2}\right)$

\begin{tabular}{lllllll}
\hline & $U^{11}$ & $U^{22}$ & $U^{33}$ & $U^{12}$ & $U^{13}$ & $U^{23}$ \\
\hline $\mathrm{C} 1 \mathrm{~S}$ & $0.059(4)$ & $0.039(3)$ & $0.060(4)$ & $-0.004(3)$ & $0.052(3)$ & $-0.005(3)$
\end{tabular}




\begin{tabular}{|c|c|c|c|c|c|c|}
\hline C3S & $0.046(4)$ & $0.037(4)$ & $0.062(5)$ & -0.005 (3) & $-0.005(3)$ & $0.022(4)$ \\
\hline $\mathrm{C} 2 \mathrm{~S}$ & 0.109 (7) & $0.050(5)$ & $0.085(5)$ & $-0.007(5)$ & $0.078(5)$ & $0.015(4)$ \\
\hline $\mathrm{C} 4 \mathrm{~S}$ & $0.066(4)$ & $0.074(4)$ & $0.078(4)$ & 0.000 & $0.036(3)$ & 0.000 \\
\hline $\mathrm{C} 7 \mathrm{~S}$ & $0.066(4)$ & $0.074(4)$ & $0.078(4)$ & 0.000 & $0.036(3)$ & 0.000 \\
\hline $\mathrm{C} 5 \mathrm{~S}$ & $0.066(4)$ & $0.074(4)$ & $0.078(4)$ & 0.000 & $0.036(3)$ & 0.000 \\
\hline C6S & $0.066(4)$ & 0.074 (4) & $0.078(4)$ & 0.000 & $0.036(3)$ & 0.000 \\
\hline $\mathrm{N} 1 \mathrm{~A}$ & $0.0287(9)$ & $0.0273(9)$ & $0.0243(8)$ & $-0.0025(7)$ & $0.0112(7)$ & $0.0023(7)$ \\
\hline $\mathrm{N} 2 \mathrm{~A}$ & $0.0268(9)$ & $0.0328(10)$ & $0.0262(9)$ & $-0.0035(7)$ & $0.0113(7)$ & $0.0045(7)$ \\
\hline O1A & $0.0303(8)$ & $0.0260(7)$ & $0.0256(7)$ & $-0.0028(6)$ & $0.0161(6)$ & $-0.0011(6)$ \\
\hline $\mathrm{O} 2 \mathrm{~A}$ & $0.0296(8)$ & $0.0298(7)$ & $0.0266(7)$ & $0.0039(6)$ & $0.0156(6)$ & $0.0052(6)$ \\
\hline $\mathrm{O} 3 \mathrm{~A}$ & 0.0394 (9) & $0.0261(8)$ & $0.0401(9)$ & $-0.0004(6)$ & $0.0095(7)$ & $0.0005(6)$ \\
\hline $\mathrm{O} 4 \mathrm{~A}$ & $0.0395(9)$ & $0.0321(8)$ & $0.0395(9)$ & $-0.0087(7)$ & 0.0099 (7) & $-0.0075(7)$ \\
\hline $\mathrm{C} 1 \mathrm{~A}$ & $0.0284(10)$ & $0.0180(9)$ & $0.0177(9)$ & $0.0004(8)$ & $0.0083(8)$ & $-0.0007(7)$ \\
\hline $\mathrm{C} 2 \mathrm{~A}$ & $0.0268(10)$ & $0.0189(9)$ & $0.0189(9)$ & $0.0003(8)$ & $0.0078(8)$ & $-0.0033(7)$ \\
\hline $\mathrm{C} 3 \mathrm{~A}$ & $0.0254(10)$ & $0.0223(9)$ & $0.0193(9)$ & $-0.0027(8)$ & $0.0096(8)$ & $-0.0042(7)$ \\
\hline $\mathrm{C} 4 \mathrm{~A}$ & 0.0305 (11) & $0.0180(9)$ & $0.0167(9)$ & $-0.0001(8)$ & $0.0084(8)$ & $0.0013(7)$ \\
\hline $\mathrm{C} 5 \mathrm{~A}$ & $0.0234(10)$ & $0.0201(9)$ & $0.0189(9)$ & $0.0010(8)$ & $0.0051(8)$ & $-0.0029(7)$ \\
\hline C6A & $0.0248(10)$ & $0.0184(9)$ & $0.0185(9)$ & $-0.0010(8)$ & $0.0077(8)$ & $-0.0042(7)$ \\
\hline C7A & $0.0254(10)$ & $0.0232(9)$ & $0.0203(9)$ & $0.0035(8)$ & $0.0108(8)$ & $-0.0012(7)$ \\
\hline C8A & $0.0306(11)$ & $0.0236(10)$ & $0.0232(10)$ & $0.0050(8)$ & $0.0093(8)$ & $-0.0013(8)$ \\
\hline C9A & $0.0461(13)$ & $0.0283(11)$ & $0.0260(10)$ & $0.0032(10)$ & $0.0134(9)$ & $-0.0042(9)$ \\
\hline $\mathrm{C} 10 \mathrm{~A}$ & $0.0439(13)$ & $0.0301(11)$ & $0.0316(11)$ & $0.0020(10)$ & $0.0135(10)$ & $-0.0079(9)$ \\
\hline $\mathrm{C} 11 \mathrm{~A}$ & $0.0441(14)$ & $0.0325(12)$ & $0.0363(12)$ & $0.0013(10)$ & $0.0146(10)$ & $-0.0039(10)$ \\
\hline $\mathrm{C} 12 \mathrm{~A}$ & $0.0467(15)$ & $0.0354(13)$ & $0.0493(14)$ & $0.0012(11)$ & $0.0193(12)$ & $0.0000(11)$ \\
\hline C13A & $0.071(2)$ & $0.0461(15)$ & $0.0641(18)$ & $0.0039(14)$ & $0.0307(15)$ & $0.0120(13)$ \\
\hline $\mathrm{C} 14 \mathrm{~A}$ & $0.0248(10)$ & $0.0267(10)$ & $0.0242(10)$ & $-0.0030(8)$ & $0.0136(8)$ & $0.0007(8)$ \\
\hline $\mathrm{C} 15 \mathrm{~A}$ & $0.0258(11)$ & $0.0306(11)$ & $0.0237(10)$ & $-0.0004(9)$ & $0.0146(8)$ & $0.0035(8)$ \\
\hline $\mathrm{C} 16 \mathrm{~A}$ & $0.0248(11)$ & $0.0306(11)$ & $0.0264(10)$ & $-0.0040(8)$ & $0.0111(8)$ & $0.0029(8)$ \\
\hline $\mathrm{C} 17 \mathrm{~A}$ & $0.0269(11)$ & $0.0288(10)$ & $0.0250(10)$ & $-0.0041(8)$ & $0.0120(8)$ & $0.0028(8)$ \\
\hline C18A & $0.0294(11)$ & $0.0308(11)$ & $0.0261(10)$ & $-0.0028(9)$ & $0.0066(9)$ & $0.0028(9)$ \\
\hline C19A & $0.0288(11)$ & $0.0274(11)$ & $0.0332(11)$ & $-0.0013(9)$ & $0.0141(9)$ & $0.0039(9)$ \\
\hline $\mathrm{C} 20 \mathrm{~A}$ & $0.0279(11)$ & $0.0320(11)$ & $0.0283(10)$ & $-0.0098(9)$ & $0.0122(9)$ & $-0.0026(9)$ \\
\hline $\mathrm{C} 21 \mathrm{~A}$ & $0.0258(11)$ & $0.0361(12)$ & $0.0280(10)$ & $-0.0090(9)$ & $0.0067(8)$ & $0.0033(9)$ \\
\hline $\mathrm{C} 22 \mathrm{~A}$ & $0.0460(14)$ & $0.0280(12)$ & 0.0467 (14) & $-0.0066(10)$ & $0.0152(11)$ & $-0.0023(10)$ \\
\hline $\mathrm{C} 23 \mathrm{~A}$ & 0.0468 (14) & $0.0305(12)$ & 0.0469 (14) & $-0.0055(10)$ & $0.0193(12)$ & $-0.0068(10)$ \\
\hline N1B & $0.0298(9)$ & $0.0257(9)$ & $0.0266(9)$ & $0.0036(7)$ & $0.0116(7)$ & $0.0022(7)$ \\
\hline $\mathrm{N} 2 \mathrm{~B}$ & $0.0333(10)$ & $0.0227(8)$ & $0.0276(9)$ & $0.0037(7)$ & $0.0146(8)$ & $0.0016(7)$ \\
\hline O1B & $0.0257(7)$ & $0.0234(7)$ & $0.0218(7)$ & $0.0003(6)$ & $0.0055(5)$ & $0.0030(5)$ \\
\hline $\mathrm{O} 2 \mathrm{~B}$ & $0.0258(7)$ & $0.0218(7)$ & $0.0213(6)$ & $0.0045(6)$ & $0.0054(5)$ & $0.0000(5)$ \\
\hline O3B & $0.0694(12)$ & $0.0234(8)$ & $0.0560(10)$ & $-0.0021(8)$ & $0.0370(9)$ & $-0.0082(7)$ \\
\hline O4B & $0.0635(12)$ & $0.0229(8)$ & $0.0643(11)$ & $-0.0067(7)$ & $0.0399(9)$ & $-0.0047(7)$ \\
\hline C1B & $0.0216(10)$ & $0.0189(9)$ & $0.0245(9)$ & $0.0012(7)$ & $0.0087(8)$ & $0.0009(7)$ \\
\hline $\mathrm{C} 2 \mathrm{~B}$ & $0.0200(10)$ & $0.0192(9)$ & $0.0234(9)$ & $-0.0052(8)$ & $0.0099(8)$ & $-0.0014(8)$ \\
\hline $\mathrm{C} 3 \mathrm{~B}$ & $0.0193(10)$ & $0.0217(9)$ & $0.0221(9)$ & $-0.0030(8)$ & $0.0050(7)$ & $0.0010(8)$ \\
\hline C4B & $0.0200(10)$ & $0.0218(9)$ & $0.0271(10)$ & $0.0007(8)$ & $0.0080(8)$ & $0.0021(8)$ \\
\hline C5B & $0.0218(10)$ & $0.0252(10)$ & $0.0220(9)$ & $0.0064(8)$ & $0.0104(8)$ & $0.0054(8)$ \\
\hline C6B & $0.0197(10)$ & $0.0213(9)$ & $0.0209(9)$ & $0.0054(7)$ & $0.0063(7)$ & $0.0007(7)$ \\
\hline C7B & $0.0232(10)$ & $0.0205(9)$ & $0.0204(9)$ & $-0.0003(8)$ & $0.0066(8)$ & $0.0005(7)$ \\
\hline
\end{tabular}




\begin{tabular}{lllllll} 
& & & & \\
C8B & $0.0286(11)$ & $0.0209(9)$ & $0.0238(10)$ & $-0.0042(8)$ & $0.0104(8)$ & $-0.0015(8)$ \\
C9B & $0.0413(13)$ & $0.0249(10)$ & $0.0245(10)$ & $-0.0014(9)$ & $0.0105(9)$ & $-0.0040(8)$ \\
C10B & $0.0369(13)$ & $0.0298(11)$ & $0.0306(11)$ & $-0.0025(9)$ & $0.0082(9)$ & $-0.0078(9)$ \\
C11B & $0.0395(13)$ & $0.0283(11)$ & $0.0374(12)$ & $-0.0021(10)$ & $0.0101(10)$ & $-0.0040(9)$ \\
C12B & $0.0415(14)$ & $0.0275(11)$ & $0.0438(13)$ & $-0.0033(9)$ & $0.0209(11)$ & $-0.0044(9)$ \\
C13B & $0.0600(18)$ & $0.0340(13)$ & $0.0639(17)$ & $0.0004(12)$ & $0.0245(14)$ & $0.0016(12)$ \\
C14B & $0.0208(10)$ & $0.0237(10)$ & $0.0227(9)$ & $0.0047(8)$ & $0.0049(8)$ & $0.0031(8)$ \\
C15B & $0.0254(11)$ & $0.0201(9)$ & $0.0199(9)$ & $0.0046(8)$ & $0.0033(8)$ & $0.0009(7)$ \\
C16B & $0.0322(11)$ & $0.0230(10)$ & $0.0263(10)$ & $0.0061(8)$ & $0.0118(9)$ & $0.0027(8)$ \\
C17B & $0.0279(11)$ & $0.0235(10)$ & $0.0259(10)$ & $0.0043(8)$ & $0.0091(8)$ & $0.0030(8)$ \\
C18B & $0.0413(13)$ & $0.0290(11)$ & $0.0331(11)$ & $0.0050(9)$ & $0.0203(10)$ & $0.0009(9)$ \\
C19B & $0.0403(13)$ & $0.0226(10)$ & $0.0293(10)$ & $0.0056(9)$ & $0.0131(9)$ & $-0.0009(8)$ \\
C20B & $0.0358(12)$ & $0.0242(10)$ & $0.0346(11)$ & $-0.0004(9)$ & $0.0131(9)$ & $0.0034(9)$ \\
C21B & $0.0400(13)$ & $0.0286(11)$ & $0.0406(12)$ & $0.0014(9)$ & $0.0247(11)$ & $0.0019(9)$ \\
C22B & $0.137(3)$ & $0.0263(14)$ & $0.119(3)$ & $-0.0099(17)$ & $0.098(3)$ & $-0.0105(16)$ \\
C23B & $0.055(4)$ & $0.027(3)$ & $0.066(6)$ & $-0.008(3)$ & $0.024(4)$ & $-0.013(3)$ \\
C22C & $0.137(3)$ & $0.0263(14)$ & $0.119(3)$ & $-0.0099(17)$ & $0.098(3)$ & $-0.0105(16)$ \\
C23C & $0.055(5)$ & $0.018(3)$ & $0.046(5)$ & $-0.002(3)$ & $0.016(4)$ & $0.000(3)$ \\
& & & & & & \\
\hline
\end{tabular}

Geometric parameters $\left(\AA,{ }^{\circ}\right)$

\begin{tabular}{llll}
\hline $\mathrm{C} 1 \mathrm{~S}-\mathrm{C} 1 \mathrm{~S}^{\mathrm{i}}$ & $1.3798(10)$ & $\mathrm{C} 22 \mathrm{~A}-\mathrm{C} 23 \mathrm{~A}$ & $1.492(3)$ \\
$\mathrm{C} 1 \mathrm{~S}-\mathrm{C} 2 \mathrm{~S}$ & $1.3803(10)$ & $\mathrm{C} 22 \mathrm{~A}-\mathrm{H} 22 \mathrm{~A}$ & 0.9900 \\
$\mathrm{C} 1 \mathrm{~S}-\mathrm{H} 1 \mathrm{~S}$ & 0.9500 & $\mathrm{C} 22 \mathrm{~A}-\mathrm{H} 22 \mathrm{~B}$ & 0.9900 \\
$\mathrm{C} 3 \mathrm{~S}-\mathrm{C} 3 \mathrm{~S}^{\mathrm{i}}$ & $1.3800(10)$ & $\mathrm{C} 23 \mathrm{~A}-\mathrm{H} 23 \mathrm{~A}$ & 0.9900 \\
$\mathrm{C} 3 \mathrm{~S}-\mathrm{C} 2 \mathrm{~S}$ & $1.3804(10)$ & $\mathrm{C} 23 \mathrm{~A}-\mathrm{H} 23 \mathrm{~B}$ & 0.9900 \\
$\mathrm{C} 3 \mathrm{~S}-\mathrm{H} 3 \mathrm{~S}$ & 0.9500 & $\mathrm{~N} 1 \mathrm{~B}-\mathrm{C} 14 \mathrm{~B}$ & $1.292(2)$ \\
$\mathrm{C} 2 \mathrm{~S}-\mathrm{H} 2 \mathrm{~S}$ & 0.9500 & $\mathrm{~N} 1 \mathrm{~B}-\mathrm{C} 17 \mathrm{~B}$ & $1.374(3)$ \\
$\mathrm{C} 4 \mathrm{~S}-\mathrm{C} 5 \mathrm{~S}^{\mathrm{i}}$ & $1.365(13)$ & $\mathrm{N} 2 \mathrm{~B}-\mathrm{C} 15 \mathrm{~B}$ & $1.290(2)$ \\
$\mathrm{C} 4 \mathrm{~S}-\mathrm{C} 5 \mathrm{~S}$ & $1.365(13)$ & $\mathrm{N} 2 \mathrm{~B}-\mathrm{C} 16 \mathrm{~B}$ & $1.377(2)$ \\
$\mathrm{C} 4 \mathrm{~S}-\mathrm{H} 4 \mathrm{~S}$ & 0.9500 & $\mathrm{O} 1 \mathrm{~B}-\mathrm{C} 14 \mathrm{~B}$ & $1.387(2)$ \\
$\mathrm{C} 7 \mathrm{~S}-\mathrm{C} 6 \mathrm{~S}$ & $1.351(10)$ & $\mathrm{O} 1 \mathrm{~B}-\mathrm{C} 3 \mathrm{~B}$ & $1.408(2)$ \\
$\mathrm{C} 7 \mathrm{~S}-\mathrm{C} 6 \mathrm{~S}^{\mathrm{i}}$ & $1.351(10)$ & $\mathrm{O} 2 \mathrm{~B}-\mathrm{C} 15 \mathrm{~B}$ & $1.383(2)$ \\
$\mathrm{C} 7 \mathrm{~S}-\mathrm{H} 7 \mathrm{~S}$ & 0.9500 & $\mathrm{O} 3 \mathrm{~B}-\mathrm{C} 19 \mathrm{~B}$ & $1.376(2)$ \\
$\mathrm{C} 5 \mathrm{~S}-\mathrm{C} 6 \mathrm{~S}$ & $1.345(11)$ & $\mathrm{O} 3 \mathrm{~B}-\mathrm{C} 22 \mathrm{C}$ & $1.386(3)$ \\
$\mathrm{C} 5 \mathrm{~S}-\mathrm{H} 5 \mathrm{~S}$ & 0.9500 & $\mathrm{O} 3 \mathrm{~B}-\mathrm{C} 22 \mathrm{~B}$ & $1.386(3)$ \\
$\mathrm{C} 6 \mathrm{~S}-\mathrm{H} 6 \mathrm{~S}$ & $\mathrm{O} 4 \mathrm{~B}-\mathrm{C} 20 \mathrm{~B}$ & $1.367(2)$ \\
$\mathrm{N} 1 \mathrm{~A}-\mathrm{C} 14 \mathrm{~A}$ & 1.9500 & $\mathrm{O} 4 \mathrm{~B}-\mathrm{C} 23 \mathrm{C}$ & $1.404(7)$ \\
$\mathrm{N} 1 \mathrm{~A}-\mathrm{C} 17 \mathrm{~A}$ & $\mathrm{O} 4 \mathrm{~B}-\mathrm{C} 23 \mathrm{~B}$ & $1.427(6)$ \\
$\mathrm{N} 2 \mathrm{~A}-\mathrm{C} 15 \mathrm{~A}$ & $\mathrm{C} 1 \mathrm{~B}-\mathrm{C} 2 \mathrm{~B}^{\mathrm{ii}}$ & $1.394(3)$ \\
$\mathrm{N} 2 \mathrm{~A}-\mathrm{C} 16 \mathrm{~A}$ & $\mathrm{C} 1 \mathrm{~B}-\mathrm{C} 6 \mathrm{~B}$ & $1.396(3)$ \\
$\mathrm{O} 1 \mathrm{~A}-\mathrm{C} 14 \mathrm{~A}$ & $\mathrm{C} 1 \mathrm{~B}-\mathrm{H} 1 \mathrm{~B}$ & 0.9500 \\
$\mathrm{O} 1 \mathrm{~A}-\mathrm{C} 3 \mathrm{~A}$ & $1.375(2)$ & $\mathrm{C} 2 \mathrm{~B}-\mathrm{C} 3 \mathrm{~B}$ & $1.386(3)$ \\
$\mathrm{O} 2 \mathrm{~A}-\mathrm{C} 15 \mathrm{~A}$ & $\mathrm{C} 2 \mathrm{~B}-\mathrm{C} 1 \mathrm{~B}^{\mathrm{ii}}$ & $1.394(3)$ \\
$\mathrm{O} 2 \mathrm{~A}-\mathrm{C} 5 \mathrm{~B}$ & $1.292(2)$ & $\mathrm{C} 2 \mathrm{~B}-\mathrm{C} 7 \mathrm{~B}$ & $1.530(2)$ \\
$\mathrm{O} 3 \mathrm{~A}-\mathrm{C} 19 \mathrm{~A}$ & $\mathrm{C} 3 \mathrm{~B}-\mathrm{C} 4 \mathrm{~B}^{\mathrm{ii}}$ & $1.385(3)$ \\
$\mathrm{O} 3 \mathrm{~A}-\mathrm{C} 22 \mathrm{~A}$ & $1.369(2)$ & $\mathrm{C} 4 \mathrm{~B}-\mathrm{C} 5 \mathrm{~B}$ & $1.381(3)$ \\
$\mathrm{O} 4 \mathrm{~A}-\mathrm{C} 20 \mathrm{~A}$ & $1.414(2)$ & $\mathrm{C} 4 \mathrm{~B}-\mathrm{C} 3 \mathrm{~B}^{\mathrm{ii}}$ & $1.384(3)$ \\
& $1.378(2)$ & &
\end{tabular}




\begin{tabular}{|c|c|c|c|}
\hline $\mathrm{O} 4 \mathrm{~A}-\mathrm{C} 23 \mathrm{~A}$ & $1.432(3)$ & $\mathrm{C} 4 \mathrm{~B}-\mathrm{H} 4 \mathrm{~B}$ & 0.9500 \\
\hline $\mathrm{C} 1 \mathrm{~A}-\mathrm{C} 2 \mathrm{~A}$ & $1.396(3)$ & $\mathrm{C} 5 \mathrm{~B}-\mathrm{C} 6 \mathrm{~B}$ & $1.390(3)$ \\
\hline $\mathrm{C} 1 \mathrm{~A}-\mathrm{C} 6 \mathrm{~A}$ & $1.397(3)$ & $\mathrm{C} 7 \mathrm{~B}-\mathrm{C} 8 \mathrm{~B}$ & $1.536(2)$ \\
\hline $\mathrm{C} 1 \mathrm{~A}-\mathrm{H} 1 \mathrm{~A}$ & 0.9500 & $\mathrm{C} 7 \mathrm{~B}-\mathrm{H} 7 \mathrm{~B}$ & 1.0000 \\
\hline $\mathrm{C} 2 \mathrm{~A}-\mathrm{C} 3 \mathrm{~A}$ & $1.396(2)$ & $\mathrm{C} 8 \mathrm{~B}-\mathrm{C} 9 \mathrm{~B}$ & $1.524(3)$ \\
\hline $\mathrm{C} 2 \mathrm{~A}-\mathrm{C} 7 \mathrm{~A}$ & $1.529(3)$ & $\mathrm{C} 8 \mathrm{~B}-\mathrm{H} 8 \mathrm{~B} 1$ & 0.9900 \\
\hline $\mathrm{C} 3 \mathrm{~A}-\mathrm{C} 4 \mathrm{~A}$ & $1.381(3)$ & $\mathrm{C} 8 \mathrm{~B}-\mathrm{H} 8 \mathrm{~B} 2$ & 0.9900 \\
\hline $\mathrm{C} 4 \mathrm{~A}-\mathrm{C} 5 \mathrm{~A}$ & $1.382(3)$ & $\mathrm{C} 9 \mathrm{~B}-\mathrm{C} 10 \mathrm{~B}$ & $1.526(3)$ \\
\hline $\mathrm{C} 4 \mathrm{~A}-\mathrm{H} 4 \mathrm{~A}$ & 0.9500 & C9B-H9B1 & 0.9900 \\
\hline $\mathrm{C} 5 \mathrm{~A}-\mathrm{C} 6 \mathrm{~A}$ & $1.393(3)$ & $\mathrm{C} 9 \mathrm{~B}-\mathrm{H} 9 \mathrm{~B} 2$ & 0.9900 \\
\hline $\mathrm{C} 5 \mathrm{~A}-\mathrm{O} 2 \mathrm{~B}$ & $1.405(2)$ & $\mathrm{C} 10 \mathrm{~B}-\mathrm{C} 11 \mathrm{~B}$ & $1.514(3)$ \\
\hline $\mathrm{C} 6 \mathrm{~A}-\mathrm{C} 7 \mathrm{~B}$ & $1.524(3)$ & $\mathrm{C} 10 \mathrm{~B}-\mathrm{H} 10 \mathrm{C}$ & 0.9900 \\
\hline $\mathrm{C} 7 \mathrm{~A}-\mathrm{C} 8 \mathrm{~A}$ & $1.531(2)$ & $\mathrm{C} 10 \mathrm{~B}-\mathrm{H} 10 \mathrm{D}$ & 0.9900 \\
\hline $\mathrm{C} 7 \mathrm{~A}-\mathrm{C} 6 \mathrm{~B}$ & $1.531(2)$ & $\mathrm{C} 11 \mathrm{~B}-\mathrm{C} 12 \mathrm{~B}$ & $1.521(3)$ \\
\hline $\mathrm{C} 7 \mathrm{~A}-\mathrm{H} 7 \mathrm{~A}$ & 1.0000 & $\mathrm{C} 11 \mathrm{~B}-\mathrm{H} 11 \mathrm{C}$ & 0.9900 \\
\hline $\mathrm{C} 8 \mathrm{~A}-\mathrm{C} 9 \mathrm{~A}$ & $1.529(3)$ & C11B-H11D & 0.9900 \\
\hline $\mathrm{C} 8 \mathrm{~A}-\mathrm{H} 8 \mathrm{~A} 1$ & 0.9900 & $\mathrm{C} 12 \mathrm{~B}-\mathrm{C} 13 \mathrm{~B}$ & $1.513(3)$ \\
\hline $\mathrm{C} 8 \mathrm{~A}-\mathrm{H} 8 \mathrm{~A} 2$ & 0.9900 & $\mathrm{C} 12 \mathrm{~B}-\mathrm{H} 12 \mathrm{C}$ & 0.9900 \\
\hline $\mathrm{C} 9 \mathrm{~A}-\mathrm{C} 10 \mathrm{~A}$ & $1.532(3)$ & $\mathrm{C} 12 \mathrm{~B}-\mathrm{H} 12 \mathrm{D}$ & 0.9900 \\
\hline C9A-H9A1 & 0.9900 & $\mathrm{C} 13 \mathrm{~B}-\mathrm{H} 13 \mathrm{D}$ & 0.9800 \\
\hline $\mathrm{C} 9 \mathrm{~A}-\mathrm{H} 9 \mathrm{~A} 2$ & 0.9900 & $\mathrm{C} 13 \mathrm{~B}-\mathrm{H} 13 \mathrm{E}$ & 0.9800 \\
\hline $\mathrm{C} 10 \mathrm{~A}-\mathrm{C} 11 \mathrm{~A}$ & $1.520(3)$ & $\mathrm{C} 13 \mathrm{~B}-\mathrm{H} 13 \mathrm{~F}$ & 0.9800 \\
\hline $\mathrm{C} 10 \mathrm{~A}-\mathrm{H} 10 \mathrm{~A}$ & 0.9900 & $\mathrm{C} 14 \mathrm{~B}-\mathrm{C} 15 \mathrm{~B}$ & $1.424(3)$ \\
\hline $\mathrm{C} 10 \mathrm{~A}-\mathrm{H} 10 \mathrm{~B}$ & 0.9900 & $\mathrm{C} 16 \mathrm{~B}-\mathrm{C} 21 \mathrm{~B}$ & $1.401(3)$ \\
\hline $\mathrm{C} 11 \mathrm{~A}-\mathrm{C} 12 \mathrm{~A}$ & $1.523(3)$ & $\mathrm{C} 16 \mathrm{~B}-\mathrm{C} 17 \mathrm{~B}$ & $1.419(3)$ \\
\hline $\mathrm{C} 11 \mathrm{~A}-\mathrm{H} 11 \mathrm{~A}$ & 0.9900 & $\mathrm{C} 17 \mathrm{~B}-\mathrm{C} 18 \mathrm{~B}$ & $1.398(3)$ \\
\hline $\mathrm{C} 11 \mathrm{~A}-\mathrm{H} 11 \mathrm{~B}$ & 0.9900 & $\mathrm{C} 18 \mathrm{~B}-\mathrm{C} 19 \mathrm{~B}$ & $1.362(3)$ \\
\hline $\mathrm{C} 12 \mathrm{~A}-\mathrm{C} 13 \mathrm{~A}$ & $1.512(3)$ & $\mathrm{C} 18 \mathrm{~B}-\mathrm{H} 18 \mathrm{~B}$ & 0.9500 \\
\hline $\mathrm{C} 12 \mathrm{~A}-\mathrm{H} 12 \mathrm{~A}$ & 0.9900 & $\mathrm{C} 19 \mathrm{~B}-\mathrm{C} 20 \mathrm{~B}$ & $1.417(3)$ \\
\hline $\mathrm{C} 12 \mathrm{~A}-\mathrm{H} 12 \mathrm{~B}$ & 0.9900 & $\mathrm{C} 20 \mathrm{~B}-\mathrm{C} 21 \mathrm{~B}$ & $1.364(3)$ \\
\hline $\mathrm{C} 13 \mathrm{~A}-\mathrm{H} 13 \mathrm{~A}$ & 0.9800 & $\mathrm{C} 21 \mathrm{~B}-\mathrm{H} 21 \mathrm{~B}$ & 0.9500 \\
\hline $\mathrm{C} 13 \mathrm{~A}-\mathrm{H} 13 \mathrm{~B}$ & 0.9800 & $\mathrm{C} 22 \mathrm{~B}-\mathrm{C} 23 \mathrm{~B}$ & $1.351(7)$ \\
\hline $\mathrm{C} 13 \mathrm{~A}-\mathrm{H} 13 \mathrm{C}$ & 0.9800 & $\mathrm{C} 22 \mathrm{~B}-\mathrm{H} 22 \mathrm{C}$ & 0.9900 \\
\hline $\mathrm{C} 14 \mathrm{~A}-\mathrm{C} 15 \mathrm{~A}$ & $1.424(3)$ & $\mathrm{C} 22 \mathrm{~B}-\mathrm{H} 22 \mathrm{D}$ & 0.9900 \\
\hline $\mathrm{C} 16 \mathrm{~A}-\mathrm{C} 21 \mathrm{~A}$ & $1.407(3)$ & $\mathrm{C} 23 \mathrm{~B}-\mathrm{H} 23 \mathrm{C}$ & 0.9900 \\
\hline $\mathrm{C} 16 \mathrm{~A}-\mathrm{C} 17 \mathrm{~A}$ & $1.417(3)$ & $\mathrm{C} 23 \mathrm{~B}-\mathrm{H} 23 \mathrm{D}$ & 0.9900 \\
\hline $\mathrm{C} 17 \mathrm{~A}-\mathrm{C} 18 \mathrm{~A}$ & $1.401(3)$ & $\mathrm{C} 22 \mathrm{C}-\mathrm{C} 23 \mathrm{C}$ & $1.418(8)$ \\
\hline $\mathrm{C} 18 \mathrm{~A}-\mathrm{C} 19 \mathrm{~A}$ & $1.366(3)$ & $\mathrm{C} 22 \mathrm{C}-\mathrm{H} 22 \mathrm{E}$ & 0.9900 \\
\hline $\mathrm{C} 18 \mathrm{~A}-\mathrm{H} 18 \mathrm{~A}$ & 0.9500 & $\mathrm{C} 22 \mathrm{C}-\mathrm{H} 22 \mathrm{~F}$ & 0.9900 \\
\hline $\mathrm{C} 19 \mathrm{~A}-\mathrm{C} 20 \mathrm{~A}$ & $1.421(3)$ & $\mathrm{C} 23 \mathrm{C}-\mathrm{H} 23 \mathrm{E}$ & 0.9900 \\
\hline $\mathrm{C} 20 \mathrm{~A}-\mathrm{C} 21 \mathrm{~A}$ & $1.364(3)$ & $\mathrm{C} 23 \mathrm{C}-\mathrm{H} 23 \mathrm{~F}$ & 0.9900 \\
\hline $\mathrm{C} 21 \mathrm{~A}-\mathrm{H} 21 \mathrm{~A}$ & 0.9500 & & \\
\hline $\mathrm{C} 22 A \cdots \mathrm{C} 23 B$ & $4.053(9)$ & $\mathrm{C} 22 A \cdots \mathrm{C} 23 A^{\mathrm{i}}$ & $8.181(4)$ \\
\hline $\mathrm{C} 23 A \cdots \mathrm{C} 22 B^{\mathrm{i}}$ & $3.757(5)$ & $\mathrm{C} 22 B \cdots \mathrm{C} 23 B^{\mathrm{i}}$ & $4.664(9)$ \\
\hline $\mathrm{C} 1 \mathrm{~S}-\mathrm{C} 1 \mathrm{~S}-\mathrm{C} 2 \mathrm{~S}$ & $119.4(2)$ & $\mathrm{O} 4 \mathrm{~A}-\mathrm{C} 23 \mathrm{~A}-\mathrm{H} 23 \mathrm{~B}$ & 109.6 \\
\hline $\mathrm{C} 1 \mathrm{~S}-\mathrm{C} 1 \mathrm{~S}-\mathrm{H} 1 \mathrm{~S}$ & 120.3 & $\mathrm{C} 22 \mathrm{~A}-\mathrm{C} 23 \mathrm{~A}-\mathrm{H} 23 \mathrm{~B}$ & 109.6 \\
\hline
\end{tabular}




\begin{tabular}{|c|c|}
\hline $\mathrm{C} 2 \mathrm{~S}-\mathrm{C} 1 \mathrm{~S}-\mathrm{H} 1 \mathrm{~S}$ & 120.3 \\
\hline $\mathrm{C} 3 \mathrm{~S}-\mathrm{C} 3 \mathrm{~S}-\mathrm{C} 2 \mathrm{~S}$ & $119.4(2)$ \\
\hline $\mathrm{C} 3 \mathrm{~S}^{\mathrm{i}}-\mathrm{C} 3 \mathrm{~S}-\mathrm{H} 3 \mathrm{~S}$ & 120.3 \\
\hline $\mathrm{C} 2 \mathrm{~S}-\mathrm{C} 3 \mathrm{~S}-\mathrm{H} 3 \mathrm{~S}$ & 120.3 \\
\hline $\mathrm{C} 1 \mathrm{~S}-\mathrm{C} 2 \mathrm{~S}-\mathrm{C} 3 \mathrm{~S}$ & $121.1(4)$ \\
\hline $\mathrm{C} 1 \mathrm{~S}-\mathrm{C} 2 \mathrm{~S}-\mathrm{H} 2 \mathrm{~S}$ & 119.4 \\
\hline $\mathrm{C} 3 \mathrm{~S}-\mathrm{C} 2 \mathrm{~S}-\mathrm{H} 2 \mathrm{~S}$ & 119.4 \\
\hline $\mathrm{C} 5 \mathrm{~S}-\mathrm{C} 4 \mathrm{~S}-\mathrm{C} 5 \mathrm{~S}$ & $118.2(16)$ \\
\hline $\mathrm{C} 5 \mathrm{~S}^{\mathrm{i}}-\mathrm{C} 4 \mathrm{~S}-\mathrm{H} 4 \mathrm{~S}$ & 120.9 \\
\hline $\mathrm{C} 5 \mathrm{~S}-\mathrm{C} 4 \mathrm{~S}-\mathrm{H} 4 \mathrm{~S}$ & 120.9 \\
\hline $\mathrm{C} 6 \mathrm{~S}-\mathrm{C} 7 \mathrm{~S}-\mathrm{C} 6 \mathrm{~S}^{\mathrm{i}}$ & $118.6(13)$ \\
\hline $\mathrm{C} 6 \mathrm{~S}-\mathrm{C} 7 \mathrm{~S}-\mathrm{H} 7 \mathrm{~S}$ & 120.7 \\
\hline $\mathrm{C} 6 \mathrm{~S}^{\mathrm{i}}-\mathrm{C} 7 \mathrm{~S}-\mathrm{H} 7 \mathrm{~S}$ & 120.7 \\
\hline $\mathrm{C} 6 \mathrm{~S}-\mathrm{C} 5 \mathrm{~S}-\mathrm{C} 4 \mathrm{~S}$ & $120.5(11)$ \\
\hline $\mathrm{C} 6 \mathrm{~S}-\mathrm{C} 5 \mathrm{~S}-\mathrm{H} 5 \mathrm{~S}$ & 119.8 \\
\hline $\mathrm{C} 4 \mathrm{~S}-\mathrm{C} 5 \mathrm{~S}-\mathrm{H} 5 \mathrm{~S}$ & 119.8 \\
\hline $\mathrm{C} 5 \mathrm{~S}-\mathrm{C} 6 \mathrm{~S}-\mathrm{C} 7 \mathrm{~S}$ & $121.1(10)$ \\
\hline $\mathrm{C} 5 \mathrm{~S}-\mathrm{C} 6 \mathrm{~S}-\mathrm{H} 6 \mathrm{~S}$ & 119.4 \\
\hline $\mathrm{C} 7 \mathrm{~S}-\mathrm{C} 6 \mathrm{~S}-\mathrm{H} 6 \mathrm{~S}$ & 119.4 \\
\hline $\mathrm{C} 14 \mathrm{~A}-\mathrm{N} 1 \mathrm{~A}-\mathrm{C} 17 \mathrm{~A}$ & $116.28(17)$ \\
\hline $\mathrm{C} 15 \mathrm{~A}-\mathrm{N} 2 \mathrm{~A}-\mathrm{C} 16 \mathrm{~A}$ & $116.06(17)$ \\
\hline $\mathrm{C} 14 \mathrm{~A}-\mathrm{O} 1 \mathrm{~A}-\mathrm{C} 3 \mathrm{~A}$ & $114.12(14)$ \\
\hline $\mathrm{C} 15 \mathrm{~A}-\mathrm{O} 2 \mathrm{~A}-\mathrm{C} 5 \mathrm{~B}$ & $113.73(14)$ \\
\hline $\mathrm{C} 19 \mathrm{~A}-\mathrm{O} 3 \mathrm{~A}-\mathrm{C} 22 \mathrm{~A}$ & $115.36(17)$ \\
\hline $\mathrm{C} 20 \mathrm{~A}-\mathrm{O} 4 \mathrm{~A}-\mathrm{C} 23 \mathrm{~A}$ & $112.77(16)$ \\
\hline $\mathrm{C} 2 \mathrm{~A}-\mathrm{C} 1 \mathrm{~A}-\mathrm{C} 6 \mathrm{~A}$ & $123.77(17)$ \\
\hline $\mathrm{C} 2 \mathrm{~A}-\mathrm{C} 1 \mathrm{~A}-\mathrm{H} 1 \mathrm{~A}$ & 118.1 \\
\hline $\mathrm{C} 6 \mathrm{~A}-\mathrm{C} 1 \mathrm{~A}-\mathrm{H} 1 \mathrm{~A}$ & 118.1 \\
\hline $\mathrm{C} 3 \mathrm{~A}-\mathrm{C} 2 \mathrm{~A}-\mathrm{C} 1 \mathrm{~A}$ & $116.25(17)$ \\
\hline $\mathrm{C} 3 \mathrm{~A}-\mathrm{C} 2 \mathrm{~A}-\mathrm{C} 7 \mathrm{~A}$ & $122.16(17)$ \\
\hline $\mathrm{C} 1 \mathrm{~A}-\mathrm{C} 2 \mathrm{~A}-\mathrm{C} 7 \mathrm{~A}$ & $121.54(16)$ \\
\hline $\mathrm{C} 4 \mathrm{~A}-\mathrm{C} 3 \mathrm{~A}-\mathrm{C} 2 \mathrm{~A}$ & $122.34(18)$ \\
\hline $\mathrm{C} 4 \mathrm{~A}-\mathrm{C} 3 \mathrm{~A}-\mathrm{O} 1 \mathrm{~A}$ & $118.68(16)$ \\
\hline $\mathrm{C} 2 \mathrm{~A}-\mathrm{C} 3 \mathrm{~A}-\mathrm{O} 1 \mathrm{~A}$ & $118.95(17)$ \\
\hline $\mathrm{C} 3 \mathrm{~A}-\mathrm{C} 4 \mathrm{~A}-\mathrm{C} 5 \mathrm{~A}$ & $118.82(17)$ \\
\hline $\mathrm{C} 3 \mathrm{~A}-\mathrm{C} 4 \mathrm{~A}-\mathrm{H} 4 \mathrm{~A}$ & 120.6 \\
\hline $\mathrm{C} 5 \mathrm{~A}-\mathrm{C} 4 \mathrm{~A}-\mathrm{H} 4 \mathrm{~A}$ & 120.6 \\
\hline $\mathrm{C} 4 \mathrm{~A}-\mathrm{C} 5 \mathrm{~A}-\mathrm{C} 6 \mathrm{~A}$ & $122.26(17)$ \\
\hline $\mathrm{C} 4 \mathrm{~A}-\mathrm{C} 5 \mathrm{~A}-\mathrm{O} 2 \mathrm{~B}$ & $119.19(16)$ \\
\hline $\mathrm{C} 6 \mathrm{~A}-\mathrm{C} 5 \mathrm{~A}-\mathrm{O} 2 \mathrm{~B}$ & $118.50(16)$ \\
\hline $\mathrm{C} 5 \mathrm{~A}-\mathrm{C} 6 \mathrm{~A}-\mathrm{C} 1 \mathrm{~A}$ & $116.44(17)$ \\
\hline $\mathrm{C} 5 \mathrm{~A}-\mathrm{C} 6 \mathrm{~A}-\mathrm{C} 7 \mathrm{~B}$ & $120.83(17)$ \\
\hline $\mathrm{C} 1 \mathrm{~A}-\mathrm{C} 6 \mathrm{~A}-\mathrm{C} 7 \mathrm{~B}$ & $122.70(16)$ \\
\hline $\mathrm{C} 2 \mathrm{~A}-\mathrm{C} 7 \mathrm{~A}-\mathrm{C} 8 \mathrm{~A}$ & $114.68(16)$ \\
\hline $\mathrm{C} 2 \mathrm{~A}-\mathrm{C} 7 \mathrm{~A}-\mathrm{C} 6 \mathrm{~B}$ & $108.00(14)$ \\
\hline $\mathrm{C} 8 \mathrm{~A}-\mathrm{C} 7 \mathrm{~A}-\mathrm{C} 6 \mathrm{~B}$ & $112.80(15)$ \\
\hline $\mathrm{C} 2 \mathrm{~A}-\mathrm{C} 7 \mathrm{~A}-\mathrm{H} 7 \mathrm{~A}$ & 107.0 \\
\hline & \\
\hline
\end{tabular}

\begin{tabular}{|c|c|}
\hline $\mathrm{H} 23 \mathrm{~A}-\mathrm{C} 23 \mathrm{~A}-\mathrm{H} 23 \mathrm{~B}$ & 108.2 \\
\hline $\mathrm{C} 14 \mathrm{~B}-\mathrm{N} 1 \mathrm{~B}-\mathrm{C} 17 \mathrm{~B}$ & $116.48(17)$ \\
\hline $\mathrm{C} 15 \mathrm{~B}-\mathrm{N} 2 \mathrm{~B}-\mathrm{C} 16 \mathrm{~B}$ & $116.41(17)$ \\
\hline $\mathrm{C} 14 \mathrm{~B}-\mathrm{O} 1 \mathrm{~B}-\mathrm{C} 3 \mathrm{~B}$ & $114.65(14)$ \\
\hline $\mathrm{C} 15 \mathrm{~B}-\mathrm{O} 2 \mathrm{~B}-\mathrm{C} 5 \mathrm{~A}$ & $114.19(14)$ \\
\hline $\mathrm{C} 19 \mathrm{~B}-\mathrm{O} 3 \mathrm{~B}-\mathrm{C} 22 \mathrm{C}$ & $115.27(19)$ \\
\hline $\mathrm{C} 19 \mathrm{~B}-\mathrm{O} 3 \mathrm{~B}-\mathrm{C} 22 \mathrm{~B}$ & 115.27 (19) \\
\hline $\mathrm{C} 20 \mathrm{~B}-\mathrm{O} 4 \mathrm{~B}-\mathrm{C} 23 \mathrm{C}$ & $114.1(4)$ \\
\hline $\mathrm{C} 20 \mathrm{~B}-\mathrm{O} 4 \mathrm{~B}-\mathrm{C} 23 \mathrm{~B}$ & $114.6(3)$ \\
\hline $\mathrm{C} 2 \mathrm{~B}^{\mathrm{ii}}-\mathrm{C} 1 \mathrm{~B}-\mathrm{C} 6 \mathrm{~B}$ & $123.22(17)$ \\
\hline $\mathrm{C} 2 \mathrm{~B}^{\mathrm{ii}}-\mathrm{C} 1 \mathrm{~B}-\mathrm{H} 1 \mathrm{~B}$ & 118.4 \\
\hline $\mathrm{C} 6 \mathrm{~B}-\mathrm{C} 1 \mathrm{~B}-\mathrm{H} 1 \mathrm{~B}$ & 118.4 \\
\hline $\mathrm{C} 3 \mathrm{~B}-\mathrm{C} 2 \mathrm{~B}-\mathrm{C} 1 \mathrm{~B}^{\mathrm{ii}}$ & $116.97(16)$ \\
\hline $\mathrm{C} 3 \mathrm{~B}-\mathrm{C} 2 \mathrm{~B}-\mathrm{C} 7 \mathrm{~B}$ & $120.59(16)$ \\
\hline $\mathrm{C} 1 \mathrm{~B}^{\mathrm{ii}}-\mathrm{C} 2 \mathrm{~B}-\mathrm{C} 7 \mathrm{~B}$ & $122.36(16)$ \\
\hline $\mathrm{C} 4 \mathrm{~B}^{\mathrm{ii}}-\mathrm{C} 3 \mathrm{~B}-\mathrm{C} 2 \mathrm{~B}$ & $122.38(17)$ \\
\hline $\mathrm{C} 4 \mathrm{~B}{ }^{\mathrm{ii}}-\mathrm{C} 3 \mathrm{~B}-\mathrm{O} 1 \mathrm{~B}$ & $118.42(16)$ \\
\hline $\mathrm{C} 2 \mathrm{~B}-\mathrm{C} 3 \mathrm{~B}-\mathrm{O} 1 \mathrm{~B}$ & $119.06(16)$ \\
\hline $\mathrm{C} 5 \mathrm{~B}-\mathrm{C} 4 \mathrm{~B}-\mathrm{C} 3 \mathrm{~B}^{\mathrm{ii}}$ & 118.09 (17) \\
\hline $\mathrm{C} 5 \mathrm{~B}-\mathrm{C} 4 \mathrm{~B}-\mathrm{H} 4 \mathrm{~B}$ & 121.0 \\
\hline $\mathrm{C} 3 \mathrm{~B}^{\mathrm{ii}}-\mathrm{C} 4 \mathrm{~B}-\mathrm{H} 4 \mathrm{~B}$ & 121.0 \\
\hline $\mathrm{C} 4 \mathrm{~B}-\mathrm{C} 5 \mathrm{~B}-\mathrm{C} 6 \mathrm{~B}$ & $122.90(17)$ \\
\hline $\mathrm{C} 4 \mathrm{~B}-\mathrm{C} 5 \mathrm{~B}-\mathrm{O} 2 \mathrm{~A}$ & $119.06(17)$ \\
\hline $\mathrm{C} 6 \mathrm{~B}-\mathrm{C} 5 \mathrm{~B}-\mathrm{O} 2 \mathrm{~A}$ & $118.03(16)$ \\
\hline $\mathrm{C} 5 \mathrm{~B}-\mathrm{C} 6 \mathrm{~B}-\mathrm{C} 1 \mathrm{~B}$ & $116.30(17)$ \\
\hline $\mathrm{C} 5 \mathrm{~B}-\mathrm{C} 6 \mathrm{~B}-\mathrm{C} 7 \mathrm{~A}$ & $121.24(16)$ \\
\hline $\mathrm{C} 1 \mathrm{~B}-\mathrm{C} 6 \mathrm{~B}-\mathrm{C} 7 \mathrm{~A}$ & $122.41(17)$ \\
\hline $\mathrm{C} 6 \mathrm{~A}-\mathrm{C} 7 \mathrm{~B}-\mathrm{C} 2 \mathrm{~B}$ & $112.28(14)$ \\
\hline $\mathrm{C} 6 \mathrm{~A}-\mathrm{C} 7 \mathrm{~B}-\mathrm{C} 8 \mathrm{~B}$ & $112.88(15)$ \\
\hline $\mathrm{C} 2 \mathrm{~B}-\mathrm{C} 7 \mathrm{~B}-\mathrm{C} 8 \mathrm{~B}$ & $113.02(15)$ \\
\hline $\mathrm{C} 6 \mathrm{~A}-\mathrm{C} 7 \mathrm{~B}-\mathrm{H} 7 \mathrm{~B}$ & 106.0 \\
\hline $\mathrm{C} 2 \mathrm{~B}-\mathrm{C} 7 \mathrm{~B}-\mathrm{H} 7 \mathrm{~B}$ & 106.0 \\
\hline $\mathrm{C} 8 \mathrm{~B}-\mathrm{C} 7 \mathrm{~B}-\mathrm{H} 7 \mathrm{~B}$ & 106.0 \\
\hline $\mathrm{C} 9 \mathrm{~B}-\mathrm{C} 8 \mathrm{~B}-\mathrm{C} 7 \mathrm{~B}$ & $112.12(15)$ \\
\hline $\mathrm{C} 9 \mathrm{~B}-\mathrm{C} 8 \mathrm{~B}-\mathrm{H} 8 \mathrm{~B} 1$ & 109.2 \\
\hline $\mathrm{C} 7 \mathrm{~B}-\mathrm{C} 8 \mathrm{~B}-\mathrm{H} 8 \mathrm{~B} 1$ & 109.2 \\
\hline $\mathrm{C} 9 \mathrm{~B}-\mathrm{C} 8 \mathrm{~B}-\mathrm{H} 8 \mathrm{~B} 2$ & 109.2 \\
\hline $\mathrm{C} 7 \mathrm{~B}-\mathrm{C} 8 \mathrm{~B}-\mathrm{H} 8 \mathrm{~B} 2$ & 109.2 \\
\hline $\mathrm{H} 8 \mathrm{~B} 1-\mathrm{C} 8 \mathrm{~B}-\mathrm{H} 8 \mathrm{~B} 2$ & 107.9 \\
\hline $\mathrm{C} 8 \mathrm{~B}-\mathrm{C} 9 \mathrm{~B}-\mathrm{C} 10 \mathrm{~B}$ & $114.01(17)$ \\
\hline $\mathrm{C} 8 \mathrm{~B}-\mathrm{C} 9 \mathrm{~B}-\mathrm{H} 9 \mathrm{~B} 1$ & 108.7 \\
\hline $\mathrm{C} 10 \mathrm{~B}-\mathrm{C} 9 \mathrm{~B}-\mathrm{H} 9 \mathrm{~B} 1$ & 108.7 \\
\hline $\mathrm{C} 8 \mathrm{~B}-\mathrm{C} 9 \mathrm{~B}-\mathrm{H} 9 \mathrm{~B} 2$ & 108.7 \\
\hline $\mathrm{C} 10 \mathrm{~B}-\mathrm{C} 9 \mathrm{~B}-\mathrm{H} 9 \mathrm{~B} 2$ & 108.7 \\
\hline $\mathrm{H} 9 \mathrm{~B} 1-\mathrm{C} 9 \mathrm{~B}-\mathrm{H} 9 \mathrm{~B} 2$ & 107.6 \\
\hline $\mathrm{C} 11 \mathrm{~B}-\mathrm{C} 10 \mathrm{~B}-\mathrm{C} 9 \mathrm{~B}$ & $115.78(18)$ \\
\hline $\mathrm{C} 11 \mathrm{~B}-\mathrm{C} 10 \mathrm{~B}-\mathrm{H} 10 \mathrm{C}$ & 108.3 \\
\hline $\mathrm{C} 9 \mathrm{~B}-\mathrm{C} 10 \mathrm{~B}-\mathrm{H} 10 \mathrm{C}$ & 108.3 \\
\hline
\end{tabular}




\begin{tabular}{|c|c|c|c|}
\hline $\mathrm{C} 6 \mathrm{~B}-\mathrm{C} 7 \mathrm{~A}-\mathrm{H} 7 \mathrm{~A}$ & 107.0 & $\mathrm{C} 11 \mathrm{~B}-\mathrm{C} 10 \mathrm{~B}-\mathrm{H} 10 \mathrm{D}$ & 108.3 \\
\hline $\mathrm{C} 9 \mathrm{~A}-\mathrm{C} 8 \mathrm{~A}-\mathrm{C} 7 \mathrm{~A}$ & $113.53(16)$ & $\mathrm{C} 9 \mathrm{~B}-\mathrm{C} 10 \mathrm{~B}-\mathrm{H} 10 \mathrm{D}$ & 108.3 \\
\hline $\mathrm{C} 9 \mathrm{~A}-\mathrm{C} 8 \mathrm{~A}-\mathrm{H} 8 \mathrm{~A} 1$ & 108.9 & $\mathrm{H} 10 \mathrm{C}-\mathrm{C} 10 \mathrm{~B}-\mathrm{H} 10 \mathrm{D}$ & 107.4 \\
\hline $\mathrm{C} 7 \mathrm{~A}-\mathrm{C} 8 \mathrm{~A}-\mathrm{H} 8 \mathrm{~A} 1$ & 108.9 & $\mathrm{C} 10 \mathrm{~B}-\mathrm{C} 11 \mathrm{~B}-\mathrm{C} 12 \mathrm{~B}$ & $112.61(18)$ \\
\hline $\mathrm{C} 9 \mathrm{~A}-\mathrm{C} 8 \mathrm{~A}-\mathrm{H} 8 \mathrm{~A} 2$ & 108.9 & $\mathrm{C} 10 \mathrm{~B}-\mathrm{C} 11 \mathrm{~B}-\mathrm{H} 11 \mathrm{C}$ & 109.1 \\
\hline $\mathrm{C} 7 \mathrm{~A}-\mathrm{C} 8 \mathrm{~A}-\mathrm{H} 8 \mathrm{~A} 2$ & 108.9 & $\mathrm{C} 12 \mathrm{~B}-\mathrm{C} 11 \mathrm{~B}-\mathrm{H} 11 \mathrm{C}$ & 109.1 \\
\hline $\mathrm{H} 8 \mathrm{~A} 1-\mathrm{C} 8 \mathrm{~A}-\mathrm{H} 8 \mathrm{~A} 2$ & 107.7 & $\mathrm{C} 10 \mathrm{~B}-\mathrm{C} 11 \mathrm{~B}-\mathrm{H} 11 \mathrm{D}$ & 109.1 \\
\hline $\mathrm{C} 8 \mathrm{~A}-\mathrm{C} 9 \mathrm{~A}-\mathrm{C} 10 \mathrm{~A}$ & $113.86(17)$ & $\mathrm{C} 12 \mathrm{~B}-\mathrm{C} 11 \mathrm{~B}-\mathrm{H} 11 \mathrm{D}$ & 109.1 \\
\hline $\mathrm{C} 8 \mathrm{~A}-\mathrm{C} 9 \mathrm{~A}-\mathrm{H} 9 \mathrm{~A} 1$ & 108.8 & $\mathrm{H} 11 \mathrm{C}-\mathrm{C} 11 \mathrm{~B}-\mathrm{H} 11 \mathrm{D}$ & 107.8 \\
\hline $\mathrm{C} 10 \mathrm{~A}-\mathrm{C} 9 \mathrm{~A}-\mathrm{H} 9 \mathrm{~A} 1$ & 108.8 & $\mathrm{C} 13 \mathrm{~B}-\mathrm{C} 12 \mathrm{~B}-\mathrm{C} 11 \mathrm{~B}$ & $113.51(19)$ \\
\hline $\mathrm{C} 8 \mathrm{~A}-\mathrm{C} 9 \mathrm{~A}-\mathrm{H} 9 \mathrm{~A} 2$ & 108.8 & $\mathrm{C} 13 \mathrm{~B}-\mathrm{C} 12 \mathrm{~B}-\mathrm{H} 12 \mathrm{C}$ & 108.9 \\
\hline $\mathrm{C} 10 \mathrm{~A}-\mathrm{C} 9 \mathrm{~A}-\mathrm{H} 9 \mathrm{~A} 2$ & 108.8 & $\mathrm{C} 11 \mathrm{~B}-\mathrm{C} 12 \mathrm{~B}-\mathrm{H} 12 \mathrm{C}$ & 108.9 \\
\hline H9A1-C9A-H9A2 & 107.7 & $\mathrm{C} 13 \mathrm{~B}-\mathrm{C} 12 \mathrm{~B}-\mathrm{H} 12 \mathrm{D}$ & 108.9 \\
\hline $\mathrm{C} 11 \mathrm{~A}-\mathrm{C} 10 \mathrm{~A}-\mathrm{C} 9 \mathrm{~A}$ & $115.26(17)$ & $\mathrm{C} 11 \mathrm{~B}-\mathrm{C} 12 \mathrm{~B}-\mathrm{H} 12 \mathrm{D}$ & 108.9 \\
\hline $\mathrm{C} 11 \mathrm{~A}-\mathrm{C} 10 \mathrm{~A}-\mathrm{H} 10 \mathrm{~A}$ & 108.5 & $\mathrm{H} 12 \mathrm{C}-\mathrm{C} 12 \mathrm{~B}-\mathrm{H} 12 \mathrm{D}$ & 107.7 \\
\hline $\mathrm{C} 9 \mathrm{~A}-\mathrm{C} 10 \mathrm{~A}-\mathrm{H} 10 \mathrm{~A}$ & 108.5 & $\mathrm{C} 12 \mathrm{~B}-\mathrm{C} 13 \mathrm{~B}-\mathrm{H} 13 \mathrm{D}$ & 109.5 \\
\hline $\mathrm{C} 11 \mathrm{~A}-\mathrm{C} 10 \mathrm{~A}-\mathrm{H} 10 \mathrm{~B}$ & 108.5 & $\mathrm{C} 12 \mathrm{~B}-\mathrm{C} 13 \mathrm{~B}-\mathrm{H} 13 \mathrm{E}$ & 109.5 \\
\hline $\mathrm{C} 9 \mathrm{~A}-\mathrm{C} 10 \mathrm{~A}-\mathrm{H} 10 \mathrm{~B}$ & 108.5 & $\mathrm{H} 13 \mathrm{D}-\mathrm{C} 13 \mathrm{~B}-\mathrm{H} 13 \mathrm{E}$ & 109.5 \\
\hline $\mathrm{H} 10 \mathrm{~A}-\mathrm{C} 10 \mathrm{~A}-\mathrm{H} 10 \mathrm{~B}$ & 107.5 & $\mathrm{C} 12 \mathrm{~B}-\mathrm{C} 13 \mathrm{~B}-\mathrm{H} 13 \mathrm{~F}$ & 109.5 \\
\hline $\mathrm{C} 10 \mathrm{~A}-\mathrm{C} 11 \mathrm{~A}-\mathrm{C} 12 \mathrm{~A}$ & $113.08(18)$ & $\mathrm{H} 13 \mathrm{D}-\mathrm{C} 13 \mathrm{~B}-\mathrm{H} 13 \mathrm{~F}$ & 109.5 \\
\hline $\mathrm{C} 10 \mathrm{~A}-\mathrm{C} 11 \mathrm{~A}-\mathrm{H} 11 \mathrm{~A}$ & 109.0 & $\mathrm{H} 13 \mathrm{E}-\mathrm{C} 13 \mathrm{~B}-\mathrm{H} 13 \mathrm{~F}$ & 109.5 \\
\hline $\mathrm{C} 12 \mathrm{~A}-\mathrm{C} 11 \mathrm{~A}-\mathrm{H} 11 \mathrm{~A}$ & 109.0 & $\mathrm{~N} 1 \mathrm{~B}-\mathrm{C} 14 \mathrm{~B}-\mathrm{O} 1 \mathrm{~B}$ & $119.48(17)$ \\
\hline $\mathrm{C} 10 \mathrm{~A}-\mathrm{C} 11 \mathrm{~A}-\mathrm{H} 11 \mathrm{~B}$ & 109.0 & $\mathrm{~N} 1 \mathrm{~B}-\mathrm{C} 14 \mathrm{~B}-\mathrm{C} 15 \mathrm{~B}$ & $122.85(18)$ \\
\hline $\mathrm{C} 12 \mathrm{~A}-\mathrm{C} 11 \mathrm{~A}-\mathrm{H} 11 \mathrm{~B}$ & 109.0 & $\mathrm{O} 1 \mathrm{~B}-\mathrm{C} 14 \mathrm{~B}-\mathrm{C} 15 \mathrm{~B}$ & $117.66(16)$ \\
\hline $\mathrm{H} 11 \mathrm{~A}-\mathrm{C} 11 \mathrm{~A}-\mathrm{H} 11 \mathrm{~B}$ & 107.8 & $\mathrm{~N} 2 \mathrm{~B}-\mathrm{C} 15 \mathrm{~B}-\mathrm{O} 2 \mathrm{~B}$ & $119.43(17)$ \\
\hline $\mathrm{C} 13 \mathrm{~A}-\mathrm{C} 12 \mathrm{~A}-\mathrm{C} 11 \mathrm{~A}$ & $113.1(2)$ & $\mathrm{N} 2 \mathrm{~B}-\mathrm{C} 15 \mathrm{~B}-\mathrm{C} 14 \mathrm{~B}$ & $122.83(17)$ \\
\hline $\mathrm{C} 13 \mathrm{~A}-\mathrm{C} 12 \mathrm{~A}-\mathrm{H} 12 \mathrm{~A}$ & 109.0 & $\mathrm{O} 2 \mathrm{~B}-\mathrm{C} 15 \mathrm{~B}-\mathrm{C} 14 \mathrm{~B}$ & $117.72(16)$ \\
\hline $\mathrm{C} 11 \mathrm{~A}-\mathrm{C} 12 \mathrm{~A}-\mathrm{H} 12 \mathrm{~A}$ & 109.0 & $\mathrm{~N} 2 \mathrm{~B}-\mathrm{C} 16 \mathrm{~B}-\mathrm{C} 21 \mathrm{~B}$ & $119.98(18)$ \\
\hline $\mathrm{C} 13 \mathrm{~A}-\mathrm{C} 12 \mathrm{~A}-\mathrm{H} 12 \mathrm{~B}$ & 109.0 & $\mathrm{~N} 2 \mathrm{~B}-\mathrm{C} 16 \mathrm{~B}-\mathrm{C} 17 \mathrm{~B}$ & $120.76(18)$ \\
\hline $\mathrm{C} 11 \mathrm{~A}-\mathrm{C} 12 \mathrm{~A}-\mathrm{H} 12 \mathrm{~B}$ & 109.0 & $\mathrm{C} 21 \mathrm{~B}-\mathrm{C} 16 \mathrm{~B}-\mathrm{C} 17 \mathrm{~B}$ & $119.26(18)$ \\
\hline $\mathrm{H} 12 \mathrm{~A}-\mathrm{C} 12 \mathrm{~A}-\mathrm{H} 12 \mathrm{~B}$ & 107.8 & $\mathrm{~N} 1 \mathrm{~B}-\mathrm{C} 17 \mathrm{~B}-\mathrm{C} 18 \mathrm{~B}$ & $120.36(18)$ \\
\hline $\mathrm{C} 12 \mathrm{~A}-\mathrm{C} 13 \mathrm{~A}-\mathrm{H} 13 \mathrm{~A}$ & 109.5 & $\mathrm{~N} 1 \mathrm{~B}-\mathrm{C} 17 \mathrm{~B}-\mathrm{C} 16 \mathrm{~B}$ & $120.66(17)$ \\
\hline $\mathrm{C} 12 \mathrm{~A}-\mathrm{C} 13 \mathrm{~A}-\mathrm{H} 13 \mathrm{~B}$ & 109.5 & $\mathrm{C} 18 \mathrm{~B}-\mathrm{C} 17 \mathrm{~B}-\mathrm{C} 16 \mathrm{~B}$ & $118.98(18)$ \\
\hline $\mathrm{H} 13 \mathrm{~A}-\mathrm{C} 13 \mathrm{~A}-\mathrm{H} 13 \mathrm{~B}$ & 109.5 & $\mathrm{C} 19 \mathrm{~B}-\mathrm{C} 18 \mathrm{~B}-\mathrm{C} 17 \mathrm{~B}$ & $120.89(19)$ \\
\hline $\mathrm{C} 12 \mathrm{~A}-\mathrm{C} 13 \mathrm{~A}-\mathrm{H} 13 \mathrm{C}$ & 109.5 & $\mathrm{C} 19 \mathrm{~B}-\mathrm{C} 18 \mathrm{~B}-\mathrm{H} 18 \mathrm{~B}$ & 119.6 \\
\hline $\mathrm{H} 13 \mathrm{~A}-\mathrm{C} 13 \mathrm{~A}-\mathrm{H} 13 \mathrm{C}$ & 109.5 & $\mathrm{C} 17 \mathrm{~B}-\mathrm{C} 18 \mathrm{~B}-\mathrm{H} 18 \mathrm{~B}$ & 119.6 \\
\hline $\mathrm{H} 13 \mathrm{~B}-\mathrm{C} 13 \mathrm{~A}-\mathrm{H} 13 \mathrm{C}$ & 109.5 & $\mathrm{C} 18 \mathrm{~B}-\mathrm{C} 19 \mathrm{~B}-\mathrm{O} 3 \mathrm{~B}$ & $118.75(19)$ \\
\hline N1A-C14A-O1A & $119.86(17)$ & $\mathrm{C} 18 \mathrm{~B}-\mathrm{C} 19 \mathrm{~B}-\mathrm{C} 20 \mathrm{~B}$ & $120.21(18)$ \\
\hline $\mathrm{N} 1 \mathrm{~A}-\mathrm{C} 14 \mathrm{~A}-\mathrm{C} 15 \mathrm{~A}$ & $122.50(18)$ & $\mathrm{O} 3 \mathrm{~B}-\mathrm{C} 19 \mathrm{~B}-\mathrm{C} 20 \mathrm{~B}$ & $121.04(18)$ \\
\hline $\mathrm{O} 1 \mathrm{~A}-\mathrm{C} 14 \mathrm{~A}-\mathrm{C} 15 \mathrm{~A}$ & $117.63(17)$ & $\mathrm{C} 21 \mathrm{~B}-\mathrm{C} 20 \mathrm{~B}-\mathrm{O} 4 \mathrm{~B}$ & $118.90(19)$ \\
\hline $\mathrm{N} 2 \mathrm{~A}-\mathrm{C} 15 \mathrm{~A}-\mathrm{O} 2 \mathrm{~A}$ & $119.40(17)$ & $\mathrm{C} 21 \mathrm{~B}-\mathrm{C} 20 \mathrm{~B}-\mathrm{C} 19 \mathrm{~B}$ & $119.84(19)$ \\
\hline $\mathrm{N} 2 \mathrm{~A}-\mathrm{C} 15 \mathrm{~A}-\mathrm{C} 14 \mathrm{~A}$ & $123.12(18)$ & $\mathrm{O} 4 \mathrm{~B}-\mathrm{C} 20 \mathrm{~B}-\mathrm{C} 19 \mathrm{~B}$ & $121.25(18)$ \\
\hline $\mathrm{O} 2 \mathrm{~A}-\mathrm{C} 15 \mathrm{~A}-\mathrm{C} 14 \mathrm{~A}$ & $117.48(18)$ & $\mathrm{C} 20 \mathrm{~B}-\mathrm{C} 21 \mathrm{~B}-\mathrm{C} 16 \mathrm{~B}$ & $120.8(2)$ \\
\hline $\mathrm{N} 2 \mathrm{~A}-\mathrm{C} 16 \mathrm{~A}-\mathrm{C} 21 \mathrm{~A}$ & $119.15(18)$ & $\mathrm{C} 20 \mathrm{~B}-\mathrm{C} 21 \mathrm{~B}-\mathrm{H} 21 \mathrm{~B}$ & 119.6 \\
\hline $\mathrm{N} 2 \mathrm{~A}-\mathrm{C} 16 \mathrm{~A}-\mathrm{C} 17 \mathrm{~A}$ & $121.17(18)$ & $\mathrm{C} 16 \mathrm{~B}-\mathrm{C} 21 \mathrm{~B}-\mathrm{H} 21 \mathrm{~B}$ & 119.6 \\
\hline $\mathrm{C} 21 \mathrm{~A}-\mathrm{C} 16 \mathrm{~A}-\mathrm{C} 17 \mathrm{~A}$ & $119.57(19)$ & $\mathrm{C} 23 \mathrm{~B}-\mathrm{C} 22 \mathrm{~B}-\mathrm{O} 3 \mathrm{~B}$ & $120.0(4)$ \\
\hline $\mathrm{N} 1 \mathrm{~A}-\mathrm{C} 17 \mathrm{~A}-\mathrm{C} 18 \mathrm{~A}$ & $119.83(17)$ & $\mathrm{C} 23 \mathrm{~B}-\mathrm{C} 22 \mathrm{~B}-\mathrm{H} 22 \mathrm{C}$ & 107.3 \\
\hline $\mathrm{N} 1 \mathrm{~A}-\mathrm{C} 17 \mathrm{~A}-\mathrm{C} 16 \mathrm{~A}$ & $120.64(18)$ & $\mathrm{O} 3 \mathrm{~B}-\mathrm{C} 22 \mathrm{~B}-\mathrm{H} 22 \mathrm{C}$ & 107.3 \\
\hline
\end{tabular}




\begin{tabular}{|c|c|}
\hline $\mathrm{C} 18 \mathrm{~A}-\mathrm{C} 17 \mathrm{~A}-\mathrm{C} 16 \mathrm{~A}$ & $119.46(18)$ \\
\hline $\mathrm{C} 19 \mathrm{~A}-\mathrm{C} 18 \mathrm{~A}-\mathrm{C} 17 \mathrm{~A}$ & $120.04(19)$ \\
\hline $\mathrm{C} 19 \mathrm{~A}-\mathrm{C} 18 \mathrm{~A}-\mathrm{H} 18 \mathrm{~A}$ & 120.0 \\
\hline $\mathrm{C} 17 \mathrm{~A}-\mathrm{C} 18 \mathrm{~A}-\mathrm{H} 18 \mathrm{~A}$ & 120.0 \\
\hline $\mathrm{C} 18 \mathrm{~A}-\mathrm{C} 19 \mathrm{~A}-\mathrm{O} 3 \mathrm{~A}$ & $118.15(18)$ \\
\hline $\mathrm{C} 18 \mathrm{~A}-\mathrm{C} 19 \mathrm{~A}-\mathrm{C} 20 \mathrm{~A}$ & $120.45(19)$ \\
\hline $\mathrm{O} 3 \mathrm{~A}-\mathrm{C} 19 \mathrm{~A}-\mathrm{C} 20 \mathrm{~A}$ & $121.38(18)$ \\
\hline $\mathrm{C} 21 \mathrm{~A}-\mathrm{C} 20 \mathrm{~A}-\mathrm{O} 4 \mathrm{~A}$ & $118.81(18)$ \\
\hline $\mathrm{C} 21 \mathrm{~A}-\mathrm{C} 20 \mathrm{~A}-\mathrm{C} 19 \mathrm{~A}$ & $120.21(18)$ \\
\hline $\mathrm{O} 4 \mathrm{~A}-\mathrm{C} 20 \mathrm{~A}-\mathrm{C} 19 \mathrm{~A}$ & $120.97(19)$ \\
\hline $\mathrm{C} 20 \mathrm{~A}-\mathrm{C} 21 \mathrm{~A}-\mathrm{C} 16 \mathrm{~A}$ & $119.97(19)$ \\
\hline $\mathrm{C} 20 \mathrm{~A}-\mathrm{C} 21 \mathrm{~A}-\mathrm{H} 21 \mathrm{~A}$ & 120.0 \\
\hline $\mathrm{C} 16 \mathrm{~A}-\mathrm{C} 21 \mathrm{~A}-\mathrm{H} 21 \mathrm{~A}$ & 120.0 \\
\hline $\mathrm{O} 3 \mathrm{~A}-\mathrm{C} 22 \mathrm{~A}-\mathrm{C} 23 \mathrm{~A}$ & $109.75(18)$ \\
\hline $\mathrm{O} 3 \mathrm{~A}-\mathrm{C} 22 \mathrm{~A}-\mathrm{H} 22 \mathrm{~A}$ & 109.7 \\
\hline $\mathrm{C} 23 \mathrm{~A}-\mathrm{C} 22 \mathrm{~A}-\mathrm{H} 22 \mathrm{~A}$ & 109.7 \\
\hline $\mathrm{O} 3 \mathrm{~A}-\mathrm{C} 22 \mathrm{~A}-\mathrm{H} 22 \mathrm{~B}$ & 109.7 \\
\hline $\mathrm{C} 23 \mathrm{~A}-\mathrm{C} 22 \mathrm{~A}-\mathrm{H} 22 \mathrm{~B}$ & 109.7 \\
\hline $\mathrm{H} 22 \mathrm{~A}-\mathrm{C} 22 \mathrm{~A}-\mathrm{H} 22 \mathrm{~B}$ & 108.2 \\
\hline $\mathrm{O} 4 \mathrm{~A}-\mathrm{C} 23 \mathrm{~A}-\mathrm{C} 22 \mathrm{~A}$ & $110.06(19)$ \\
\hline $\mathrm{O} 4 \mathrm{~A}-\mathrm{C} 23 \mathrm{~A}-\mathrm{H} 23 \mathrm{~A}$ & 109.6 \\
\hline $\mathrm{C} 22 \mathrm{~A}-\mathrm{C} 23 \mathrm{~A}-\mathrm{H} 23 \mathrm{~A}$ & 109.6 \\
\hline $\mathrm{C} 1 \mathrm{~S}-\mathrm{C} 1 \mathrm{~S}-\mathrm{C} 2 \mathrm{~S}-\mathrm{C} 3 \mathrm{~S}$ & $0.4(11)$ \\
\hline $\mathrm{C} 3 \mathrm{~S}-\mathrm{C} 3 \mathrm{~S}-\mathrm{C} 2 \mathrm{~S}-\mathrm{C} 1 \mathrm{~S}$ & $0.2(11)$ \\
\hline $\mathrm{C} 5 \mathrm{~S}^{\mathrm{i}}-\mathrm{C} 4 \mathrm{~S}-\mathrm{C} 5 \mathrm{~S}-\mathrm{C} 6 \mathrm{~S}$ & $-0.6(8)$ \\
\hline $\mathrm{C} 4 \mathrm{~S}-\mathrm{C} 5 \mathrm{~S}-\mathrm{C} 6 \mathrm{~S}-\mathrm{C} 7 \mathrm{~S}$ & $1.3(16)$ \\
\hline $\mathrm{C} 6 \mathrm{~S}-\mathrm{C} 7 \mathrm{~S}-\mathrm{C} 6 \mathrm{~S}-\mathrm{C} 5 \mathrm{~S}$ & $-0.6(8)$ \\
\hline $\mathrm{C} 6 \mathrm{~A}-\mathrm{C} 1 \mathrm{~A}-\mathrm{C} 2 \mathrm{~A}-\mathrm{C} 3 \mathrm{~A}$ & $-0.8(3)$ \\
\hline $\mathrm{C} 6 \mathrm{~A}-\mathrm{C} 1 \mathrm{~A}-\mathrm{C} 2 \mathrm{~A}-\mathrm{C} 7 \mathrm{~A}$ & $176.41(16)$ \\
\hline $\mathrm{C} 1 \mathrm{~A}-\mathrm{C} 2 \mathrm{~A}-\mathrm{C} 3 \mathrm{~A}-\mathrm{C} 4 \mathrm{~A}$ & $2.6(3)$ \\
\hline $\mathrm{C} 7 \mathrm{~A}-\mathrm{C} 2 \mathrm{~A}-\mathrm{C} 3 \mathrm{~A}-\mathrm{C} 4 \mathrm{~A}$ & $-174.63(16)$ \\
\hline $\mathrm{C} 1 \mathrm{~A}-\mathrm{C} 2 \mathrm{~A}-\mathrm{C} 3 \mathrm{~A}-\mathrm{O} 1 \mathrm{~A}$ & $-175.70(15)$ \\
\hline $\mathrm{C} 7 \mathrm{~A}-\mathrm{C} 2 \mathrm{~A}-\mathrm{C} 3 \mathrm{~A}-\mathrm{O} 1 \mathrm{~A}$ & $7.1(2)$ \\
\hline $\mathrm{C} 14 \mathrm{~A}-\mathrm{O} 1 \mathrm{~A}-\mathrm{C} 3 \mathrm{~A}-\mathrm{C} 4 \mathrm{~A}$ & $69.6(2)$ \\
\hline $\mathrm{C} 14 \mathrm{~A}-\mathrm{O} 1 \mathrm{~A}-\mathrm{C} 3 \mathrm{~A}-\mathrm{C} 2 \mathrm{~A}$ & $-112.00(18)$ \\
\hline $\mathrm{C} 2 \mathrm{~A}-\mathrm{C} 3 \mathrm{~A}-\mathrm{C} 4 \mathrm{~A}-\mathrm{C} 5 \mathrm{~A}$ & $-1.3(3)$ \\
\hline $\mathrm{O} 1 \mathrm{~A}-\mathrm{C} 3 \mathrm{~A}-\mathrm{C} 4 \mathrm{~A}-\mathrm{C} 5 \mathrm{~A}$ & $177.00(15)$ \\
\hline $\mathrm{C} 3 \mathrm{~A}-\mathrm{C} 4 \mathrm{~A}-\mathrm{C} 5 \mathrm{~A}-\mathrm{C} 6 \mathrm{~A}$ & $-1.9(3)$ \\
\hline $\mathrm{C} 3 \mathrm{~A}-\mathrm{C} 4 \mathrm{~A}-\mathrm{C} 5 \mathrm{~A}-\mathrm{O} 2 \mathrm{~B}$ & $-179.48(15)$ \\
\hline $\mathrm{C} 4 \mathrm{~A}-\mathrm{C} 5 \mathrm{~A}-\mathrm{C} 6 \mathrm{~A}-\mathrm{C} 1 \mathrm{~A}$ & $3.5(3)$ \\
\hline $\mathrm{O} 2 \mathrm{~B}-\mathrm{C} 5 \mathrm{~A}-\mathrm{C} 6 \mathrm{~A}-\mathrm{C} 1 \mathrm{~A}$ & $-178.92(15)$ \\
\hline $\mathrm{C} 4 \mathrm{~A}-\mathrm{C} 5 \mathrm{~A}-\mathrm{C} 6 \mathrm{~A}-\mathrm{C} 7 \mathrm{~B}$ & $-174.61(16)$ \\
\hline $\mathrm{O} 2 \mathrm{~B}-\mathrm{C} 5 \mathrm{~A}-\mathrm{C} 6 \mathrm{~A}-\mathrm{C} 7 \mathrm{~B}$ & $3.0(2)$ \\
\hline $\mathrm{C} 2 \mathrm{~A}-\mathrm{C} 1 \mathrm{~A}-\mathrm{C} 6 \mathrm{~A}-\mathrm{C} 5 \mathrm{~A}$ & $-2.1(3)$ \\
\hline $\mathrm{C} 2 \mathrm{~A}-\mathrm{C} 1 \mathrm{~A}-\mathrm{C} 6 \mathrm{~A}-\mathrm{C} 7 \mathrm{~B}$ & $175.98(16)$ \\
\hline $\mathrm{C} 3 \mathrm{~A}-\mathrm{C} 2 \mathrm{~A}-\mathrm{C} 7 \mathrm{~A}-\mathrm{C} 8 \mathrm{~A}$ & $-143.06(17)$ \\
\hline $\mathrm{C} 1 \mathrm{~A}-\mathrm{C} 2 \mathrm{~A}-\mathrm{C} 7 \mathrm{~A}-\mathrm{C} 8 \mathrm{~A}$ & $39.9(2)$ \\
\hline
\end{tabular}

107.3

107.3

106.9

$118.0(5)$

107.8

107.8

107.8

107.8

107.2

$121.8(4)$

106.9

106.9

106.9

106.9

106.7

$115.0(5)$

108.5

108.5

108.5

108.5

107.5

$-2.4(3)$

80.5 (2)

$-103.66(19)$

$2.2(3)$

$-176.44(16)$

$-67.5(2)$

$113.82(18)$

$-3.3(3)$

$175.37(15)$

$174.37(17)$

$-7.0(2)$

0.9 (3)

$-176.66(16)$

$-89.2(2)$

143.00 (17)

88.3 (2)

$-39.5(2)$

-95.5 (2)

86.5 (2)

135.34 (17)

-42.7 (2)

94.9 (2)

-88.4 (2)

$-135.96(18)$

40.7 (2) 


\begin{tabular}{|c|c|c|c|}
\hline $\mathrm{C} 3 \mathrm{~A}-\mathrm{C} 2 \mathrm{~A}-\mathrm{C} 7 \mathrm{~A}-\mathrm{C} 6 \mathrm{~B}$ & $90.2(2)$ & $\mathrm{C} 6 \mathrm{~A}-\mathrm{C} 7 \mathrm{~B}-\mathrm{C} 8 \mathrm{~B}-\mathrm{C} 9 \mathrm{~B}$ & $-60.6(2)$ \\
\hline $\mathrm{C} 1 \mathrm{~A}-\mathrm{C} 2 \mathrm{~A}-\mathrm{C} 7 \mathrm{~A}-\mathrm{C} 6 \mathrm{~B}$ & $-86.8(2)$ & $\mathrm{C} 2 \mathrm{~B}-\mathrm{C} 7 \mathrm{~B}-\mathrm{C} 8 \mathrm{~B}-\mathrm{C} 9 \mathrm{~B}$ & $170.66(16)$ \\
\hline $\mathrm{C} 2 \mathrm{~A}-\mathrm{C} 7 \mathrm{~A}-\mathrm{C} 8 \mathrm{~A}-\mathrm{C} 9 \mathrm{~A}$ & $55.5(2)$ & $\mathrm{C} 7 \mathrm{~B}-\mathrm{C} 8 \mathrm{~B}-\mathrm{C} 9 \mathrm{~B}-\mathrm{C} 10 \mathrm{~B}$ & $-162.95(17)$ \\
\hline $\mathrm{C} 6 \mathrm{~B}-\mathrm{C} 7 \mathrm{~A}-\mathrm{C} 8 \mathrm{~A}-\mathrm{C} 9 \mathrm{~A}$ & $179.72(16)$ & $\mathrm{C} 8 \mathrm{~B}-\mathrm{C} 9 \mathrm{~B}-\mathrm{C} 10 \mathrm{~B}-\mathrm{C} 11 \mathrm{~B}$ & $-64.0(3)$ \\
\hline $\mathrm{C} 7 \mathrm{~A}-\mathrm{C} 8 \mathrm{~A}-\mathrm{C} 9 \mathrm{~A}-\mathrm{C} 10 \mathrm{~A}$ & $163.16(18)$ & $\mathrm{C} 9 \mathrm{~B}-\mathrm{C} 10 \mathrm{~B}-\mathrm{C} 11 \mathrm{~B}-\mathrm{C} 12 \mathrm{~B}$ & $-178.55(18)$ \\
\hline $\mathrm{C} 8 \mathrm{~A}-\mathrm{C} 9 \mathrm{~A}-\mathrm{C} 10 \mathrm{~A}-\mathrm{C} 11 \mathrm{~A}$ & $52.5(3)$ & $\mathrm{C} 10 \mathrm{~B}-\mathrm{C} 11 \mathrm{~B}-\mathrm{C} 12 \mathrm{~B}-\mathrm{C} 13 \mathrm{~B}$ & $-178.6(2)$ \\
\hline $\mathrm{C} 9 \mathrm{~A}-\mathrm{C} 10 \mathrm{~A}-\mathrm{C} 11 \mathrm{~A}-\mathrm{C} 12 \mathrm{~A}$ & $172.89(19)$ & $\mathrm{C} 17 \mathrm{~B}-\mathrm{N} 1 \mathrm{~B}-\mathrm{C} 14 \mathrm{~B}-\mathrm{O} 1 \mathrm{~B}$ & $-179.37(16)$ \\
\hline $\mathrm{C} 10 \mathrm{~A}-\mathrm{C} 11 \mathrm{~A}-\mathrm{C} 12 \mathrm{~A}-\mathrm{C} 13 \mathrm{~A}$ & $174.4(2)$ & $\mathrm{C} 17 \mathrm{~B}-\mathrm{N} 1 \mathrm{~B}-\mathrm{C} 14 \mathrm{~B}-\mathrm{C} 15 \mathrm{~B}$ & $-0.7(3)$ \\
\hline $\mathrm{C} 17 \mathrm{~A}-\mathrm{N} 1 \mathrm{~A}-\mathrm{C} 14 \mathrm{~A}-\mathrm{O} 1 \mathrm{~A}$ & $175.95(16)$ & $\mathrm{C} 3 \mathrm{~B}-\mathrm{O} 1 \mathrm{~B}-\mathrm{C} 14 \mathrm{~B}-\mathrm{N} 1 \mathrm{~B}$ & $-76.9(2)$ \\
\hline $\mathrm{C} 17 \mathrm{~A}-\mathrm{N} 1 \mathrm{~A}-\mathrm{C} 14 \mathrm{~A}-\mathrm{C} 15 \mathrm{~A}$ & $-4.3(3)$ & $\mathrm{C} 3 \mathrm{~B}-\mathrm{O} 1 \mathrm{~B}-\mathrm{C} 14 \mathrm{~B}-\mathrm{C} 15 \mathrm{~B}$ & $104.30(19)$ \\
\hline $\mathrm{C} 3 \mathrm{~A}-\mathrm{O} 1 \mathrm{~A}-\mathrm{C} 14 \mathrm{~A}-\mathrm{N} 1 \mathrm{~A}$ & $-82.1(2)$ & $\mathrm{C} 16 \mathrm{~B}-\mathrm{N} 2 \mathrm{~B}-\mathrm{C} 15 \mathrm{~B}-\mathrm{O} 2 \mathrm{~B}$ & $179.13(16)$ \\
\hline $\mathrm{C} 3 \mathrm{~A}-\mathrm{O} 1 \mathrm{~A}-\mathrm{C} 14 \mathrm{~A}-\mathrm{C} 15 \mathrm{~A}$ & $98.2(2)$ & $\mathrm{C} 16 \mathrm{~B}-\mathrm{N} 2 \mathrm{~B}-\mathrm{C} 15 \mathrm{~B}-\mathrm{C} 14 \mathrm{~B}$ & $0.7(3)$ \\
\hline $\mathrm{C} 16 \mathrm{~A}-\mathrm{N} 2 \mathrm{~A}-\mathrm{C} 15 \mathrm{~A}-\mathrm{O} 2 \mathrm{~A}$ & $-177.38(16)$ & $\mathrm{C} 5 \mathrm{~A}-\mathrm{O} 2 \mathrm{~B}-\mathrm{C} 15 \mathrm{~B}-\mathrm{N} 2 \mathrm{~B}$ & $75.9(2)$ \\
\hline $\mathrm{C} 16 \mathrm{~A}-\mathrm{N} 2 \mathrm{~A}-\mathrm{C} 15 \mathrm{~A}-\mathrm{C} 14 \mathrm{~A}$ & $2.5(3)$ & $\mathrm{C} 5 \mathrm{~A}-\mathrm{O} 2 \mathrm{~B}-\mathrm{C} 15 \mathrm{~B}-\mathrm{C} 14 \mathrm{~B}$ & $-105.54(18)$ \\
\hline $\mathrm{C} 5 \mathrm{~B}-\mathrm{O} 2 \mathrm{~A}-\mathrm{C} 15 \mathrm{~A}-\mathrm{N} 2 \mathrm{~A}$ & $76.9(2)$ & $\mathrm{N} 1 \mathrm{~B}-\mathrm{C} 14 \mathrm{~B}-\mathrm{C} 15 \mathrm{~B}-\mathrm{N} 2 \mathrm{~B}$ & $0.4(3)$ \\
\hline $\mathrm{C} 5 \mathrm{~B}-\mathrm{O} 2 \mathrm{~A}-\mathrm{C} 15 \mathrm{~A}-\mathrm{C} 14 \mathrm{~A}$ & $-103.00(19)$ & $\mathrm{O} 1 \mathrm{~B}-\mathrm{C} 14 \mathrm{~B}-\mathrm{C} 15 \mathrm{~B}-\mathrm{N} 2 \mathrm{~B}$ & $179.08(16)$ \\
\hline $\mathrm{N} 1 \mathrm{~A}-\mathrm{C} 14 \mathrm{~A}-\mathrm{C} 15 \mathrm{~A}-\mathrm{N} 2 \mathrm{~A}$ & $2.1(3)$ & $\mathrm{N} 1 \mathrm{~B}-\mathrm{C} 14 \mathrm{~B}-\mathrm{C} 15 \mathrm{~B}-\mathrm{O} 2 \mathrm{~B}$ & $-178.14(16)$ \\
\hline $\mathrm{O} 1 \mathrm{~A}-\mathrm{C} 14 \mathrm{~A}-\mathrm{C} 15 \mathrm{~A}-\mathrm{N} 2 \mathrm{~A}$ & $-178.18(16)$ & $\mathrm{O} 1 \mathrm{~B}-\mathrm{C} 14 \mathrm{~B}-\mathrm{C} 15 \mathrm{~B}-\mathrm{O} 2 \mathrm{~B}$ & $0.6(2)$ \\
\hline $\mathrm{N} 1 \mathrm{~A}-\mathrm{C} 14 \mathrm{~A}-\mathrm{C} 15 \mathrm{~A}-\mathrm{O} 2 \mathrm{~A}$ & $-178.04(16)$ & $\mathrm{C} 15 \mathrm{~B}-\mathrm{N} 2 \mathrm{~B}-\mathrm{C} 16 \mathrm{~B}-\mathrm{C} 21 \mathrm{~B}$ & $178.51(19)$ \\
\hline $\mathrm{O} 1 \mathrm{~A}-\mathrm{C} 14 \mathrm{~A}-\mathrm{C} 15 \mathrm{~A}-\mathrm{O} 2 \mathrm{~A}$ & $1.7(3)$ & $\mathrm{C} 15 \mathrm{~B}-\mathrm{N} 2 \mathrm{~B}-\mathrm{C} 16 \mathrm{~B}-\mathrm{C} 17 \mathrm{~B}$ & $-1.3(3)$ \\
\hline $\mathrm{C} 15 \mathrm{~A}-\mathrm{N} 2 \mathrm{~A}-\mathrm{C} 16 \mathrm{~A}-\mathrm{C} 21 \mathrm{~A}$ & $171.71(18)$ & $\mathrm{C} 14 \mathrm{~B}-\mathrm{N} 1 \mathrm{~B}-\mathrm{C} 17 \mathrm{~B}-\mathrm{C} 18 \mathrm{~B}$ & $179.37(18)$ \\
\hline $\mathrm{C} 15 \mathrm{~A}-\mathrm{N} 2 \mathrm{~A}-\mathrm{C} 16 \mathrm{~A}-\mathrm{C} 17 \mathrm{~A}$ & $-4.4(3)$ & $\mathrm{C} 14 \mathrm{~B}-\mathrm{N} 1 \mathrm{~B}-\mathrm{C} 17 \mathrm{~B}-\mathrm{C} 16 \mathrm{~B}$ & $0.0(3)$ \\
\hline $\mathrm{C} 14 \mathrm{~A}-\mathrm{N} 1 \mathrm{~A}-\mathrm{C} 17 \mathrm{~A}-\mathrm{C} 18 \mathrm{~A}$ & $-174.73(18)$ & $\mathrm{N} 2 \mathrm{~B}-\mathrm{C} 16 \mathrm{~B}-\mathrm{C} 17 \mathrm{~B}-\mathrm{N} 1 \mathrm{~B}$ & $1.0(3)$ \\
\hline $\mathrm{C} 14 \mathrm{~A}-\mathrm{N} 1 \mathrm{~A}-\mathrm{C} 17 \mathrm{~A}-\mathrm{C} 16 \mathrm{~A}$ & $2.2(3)$ & $\mathrm{C} 21 \mathrm{~B}-\mathrm{C} 16 \mathrm{~B}-\mathrm{C} 17 \mathrm{~B}-\mathrm{N} 1 \mathrm{~B}$ & $-178.80(18)$ \\
\hline $\mathrm{N} 2 \mathrm{~A}-\mathrm{C} 16 \mathrm{~A}-\mathrm{C} 17 \mathrm{~A}-\mathrm{N} 1 \mathrm{~A}$ & $2.2(3)$ & $\mathrm{N} 2 \mathrm{~B}-\mathrm{C} 16 \mathrm{~B}-\mathrm{C} 17 \mathrm{~B}-\mathrm{C} 18 \mathrm{~B}$ & $-178.35(18)$ \\
\hline $\mathrm{C} 21 \mathrm{~A}-\mathrm{C} 16 \mathrm{~A}-\mathrm{C} 17 \mathrm{~A}-\mathrm{N} 1 \mathrm{~A}$ & $-173.92(17)$ & $\mathrm{C} 21 \mathrm{~B}-\mathrm{C} 16 \mathrm{~B}-\mathrm{C} 17 \mathrm{~B}-\mathrm{C} 18 \mathrm{~B}$ & $1.8(3)$ \\
\hline $\mathrm{N} 2 \mathrm{~A}-\mathrm{C} 16 \mathrm{~A}-\mathrm{C} 17 \mathrm{~A}-\mathrm{C} 18 \mathrm{~A}$ & $179.21(18)$ & $\mathrm{N} 1 \mathrm{~B}-\mathrm{C} 17 \mathrm{~B}-\mathrm{C} 18 \mathrm{~B}-\mathrm{C} 19 \mathrm{~B}$ & $-179.76(19)$ \\
\hline $\mathrm{C} 21 \mathrm{~A}-\mathrm{C} 16 \mathrm{~A}-\mathrm{C} 17 \mathrm{~A}-\mathrm{C} 18 \mathrm{~A}$ & $3.1(3)$ & $\mathrm{C} 16 \mathrm{~B}-\mathrm{C} 17 \mathrm{~B}-\mathrm{C} 18 \mathrm{~B}-\mathrm{C} 19 \mathrm{~B}$ & $-0.4(3)$ \\
\hline $\mathrm{N} 1 \mathrm{~A}-\mathrm{C} 17 \mathrm{~A}-\mathrm{C} 18 \mathrm{~A}-\mathrm{C} 19 \mathrm{~A}$ & $173.10(18)$ & $\mathrm{C} 17 \mathrm{~B}-\mathrm{C} 18 \mathrm{~B}-\mathrm{C} 19 \mathrm{~B}-\mathrm{O} 3 \mathrm{~B}$ & $177.87(19)$ \\
\hline $\mathrm{C} 16 \mathrm{~A}-\mathrm{C} 17 \mathrm{~A}-\mathrm{C} 18 \mathrm{~A}-\mathrm{C} 19 \mathrm{~A}$ & $-3.9(3)$ & $\mathrm{C} 17 \mathrm{~B}-\mathrm{C} 18 \mathrm{~B}-\mathrm{C} 19 \mathrm{~B}-\mathrm{C} 20 \mathrm{~B}$ & $-1.3(3)$ \\
\hline $\mathrm{C} 17 \mathrm{~A}-\mathrm{C} 18 \mathrm{~A}-\mathrm{C} 19 \mathrm{~A}-\mathrm{O} 3 \mathrm{~A}$ & $-178.31(18)$ & $\mathrm{C} 22 \mathrm{C}-\mathrm{O} 3 \mathrm{~B}-\mathrm{C} 19 \mathrm{~B}-\mathrm{C} 18 \mathrm{~B}$ & $-174.2(3)$ \\
\hline $\mathrm{C} 17 \mathrm{~A}-\mathrm{C} 18 \mathrm{~A}-\mathrm{C} 19 \mathrm{~A}-\mathrm{C} 20 \mathrm{~A}$ & $0.2(3)$ & $\mathrm{C} 22 \mathrm{~B}-\mathrm{O} 3 \mathrm{~B}-\mathrm{C} 19 \mathrm{~B}-\mathrm{C} 18 \mathrm{~B}$ & $-174.2(3)$ \\
\hline $\mathrm{C} 22 \mathrm{~A}-\mathrm{O} 3 \mathrm{~A}-\mathrm{C} 19 \mathrm{~A}-\mathrm{C} 18 \mathrm{~A}$ & $-172.14(19)$ & $\mathrm{C} 22 \mathrm{C}-\mathrm{O} 3 \mathrm{~B}-\mathrm{C} 19 \mathrm{~B}-\mathrm{C} 20 \mathrm{~B}$ & $5.0(3)$ \\
\hline $\mathrm{C} 22 \mathrm{~A}-\mathrm{O} 3 \mathrm{~A}-\mathrm{C} 19 \mathrm{~A}-\mathrm{C} 20 \mathrm{~A}$ & $9.4(3)$ & $\mathrm{C} 22 \mathrm{~B}-\mathrm{O} 3 \mathrm{~B}-\mathrm{C} 19 \mathrm{~B}-\mathrm{C} 20 \mathrm{~B}$ & $5.0(3)$ \\
\hline $\mathrm{C} 23 \mathrm{~A}-\mathrm{O} 4 \mathrm{~A}-\mathrm{C} 20 \mathrm{~A}-\mathrm{C} 21 \mathrm{~A}$ & $-160.78(19)$ & $\mathrm{C} 23 \mathrm{C}-\mathrm{O} 4 \mathrm{~B}-\mathrm{C} 20 \mathrm{~B}-\mathrm{C} 21 \mathrm{~B}$ & $155.0(6)$ \\
\hline $\mathrm{C} 23 \mathrm{~A}-\mathrm{O} 4 \mathrm{~A}-\mathrm{C} 20 \mathrm{~A}-\mathrm{C} 19 \mathrm{~A}$ & $20.2(3)$ & $\mathrm{C} 23 \mathrm{~B}-\mathrm{O} 4 \mathrm{~B}-\mathrm{C} 20 \mathrm{~B}-\mathrm{C} 21 \mathrm{~B}$ & $-170.7(6)$ \\
\hline $\mathrm{C} 18 \mathrm{~A}-\mathrm{C} 19 \mathrm{~A}-\mathrm{C} 20 \mathrm{~A}-\mathrm{C} 21 \mathrm{~A}$ & $4.6(3)$ & $\mathrm{C} 23 \mathrm{C}-\mathrm{O} 4 \mathrm{~B}-\mathrm{C} 20 \mathrm{~B}-\mathrm{C} 19 \mathrm{~B}$ & $-25.4(6)$ \\
\hline $\mathrm{O} 3 \mathrm{~A}-\mathrm{C} 19 \mathrm{~A}-\mathrm{C} 20 \mathrm{~A}-\mathrm{C} 21 \mathrm{~A}$ & $-176.99(18)$ & $\mathrm{C} 23 \mathrm{~B}-\mathrm{O} 4 \mathrm{~B}-\mathrm{C} 20 \mathrm{~B}-\mathrm{C} 19 \mathrm{~B}$ & $8.9(6)$ \\
\hline $\mathrm{C} 18 \mathrm{~A}-\mathrm{C} 19 \mathrm{~A}-\mathrm{C} 20 \mathrm{~A}-\mathrm{O} 4 \mathrm{~A}$ & $-176.38(18)$ & $\mathrm{C} 18 \mathrm{~B}-\mathrm{C} 19 \mathrm{~B}-\mathrm{C} 20 \mathrm{~B}-\mathrm{C} 21 \mathrm{~B}$ & $1.6(3)$ \\
\hline $\mathrm{O} 3 \mathrm{~A}-\mathrm{C} 19 \mathrm{~A}-\mathrm{C} 20 \mathrm{~A}-\mathrm{O} 4 \mathrm{~A}$ & $2.0(3)$ & $\mathrm{O} 3 \mathrm{~B}-\mathrm{C} 19 \mathrm{~B}-\mathrm{C} 20 \mathrm{~B}-\mathrm{C} 21 \mathrm{~B}$ & $-177.6(2)$ \\
\hline $\mathrm{O} 4 \mathrm{~A}-\mathrm{C} 20 \mathrm{~A}-\mathrm{C} 21 \mathrm{~A}-\mathrm{C} 16 \mathrm{~A}$ & $175.55(17)$ & $\mathrm{C} 18 \mathrm{~B}-\mathrm{C} 19 \mathrm{~B}-\mathrm{C} 20 \mathrm{~B}-\mathrm{O} 4 \mathrm{~B}$ & $-178.1(2)$ \\
\hline $\mathrm{C} 19 \mathrm{~A}-\mathrm{C} 20 \mathrm{~A}-\mathrm{C} 21 \mathrm{~A}-\mathrm{C} 16 \mathrm{~A}$ & $-5.4(3)$ & $\mathrm{O} 3 \mathrm{~B}-\mathrm{C} 19 \mathrm{~B}-\mathrm{C} 20 \mathrm{~B}-\mathrm{O} 4 \mathrm{~B}$ & $2.8(3)$ \\
\hline $\mathrm{N} 2 \mathrm{~A}-\mathrm{C} 16 \mathrm{~A}-\mathrm{C} 21 \mathrm{~A}-\mathrm{C} 20 \mathrm{~A}$ & $-174.61(18)$ & $\mathrm{O} 4 \mathrm{~B}-\mathrm{C} 20 \mathrm{~B}-\mathrm{C} 21 \mathrm{~B}-\mathrm{C} 16 \mathrm{~B}$ & $179.54(19)$ \\
\hline $\mathrm{C} 17 \mathrm{~A}-\mathrm{C} 16 \mathrm{~A}-\mathrm{C} 21 \mathrm{~A}-\mathrm{C} 20 \mathrm{~A}$ & $1.6(3)$ & $\mathrm{C} 19 \mathrm{~B}-\mathrm{C} 20 \mathrm{~B}-\mathrm{C} 21 \mathrm{~B}-\mathrm{C} 16 \mathrm{~B}$ & $-0.1(3)$ \\
\hline $\mathrm{C} 19 \mathrm{~A}-\mathrm{O} 3 \mathrm{~A}-\mathrm{C} 22 \mathrm{~A}-\mathrm{C} 23 \mathrm{~A}$ & $-40.4(3)$ & $\mathrm{N} 2 \mathrm{~B}-\mathrm{C} 16 \mathrm{~B}-\mathrm{C} 21 \mathrm{~B}-\mathrm{C} 20 \mathrm{~B}$ & $178.6(2)$ \\
\hline $\mathrm{C} 20 \mathrm{~A}-\mathrm{O} 4 \mathrm{~A}-\mathrm{C} 23 \mathrm{~A}-\mathrm{C} 22 \mathrm{~A}$ & $-51.6(2)$ & $\mathrm{C} 17 \mathrm{~B}-\mathrm{C} 16 \mathrm{~B}-\mathrm{C} 21 \mathrm{~B}-\mathrm{C} 20 \mathrm{~B}$ & $-1.6(3)$ \\
\hline $\mathrm{O} 3 \mathrm{~A}-\mathrm{C} 22 \mathrm{~A}-\mathrm{C} 23 \mathrm{~A}-\mathrm{O} 4 \mathrm{~A}$ & $62.5(2)$ & $\mathrm{C} 19 \mathrm{~B}-\mathrm{O} 3 \mathrm{~B}-\mathrm{C} 22 \mathrm{~B}-\mathrm{C} 23 \mathrm{~B}$ & $-26.1(8)$ \\
\hline $\mathrm{C} 4 \mathrm{~A}-\mathrm{C} 5 \mathrm{~A}-\mathrm{O} 2 \mathrm{~B}-\mathrm{C} 15 \mathrm{~B}$ & $-78.6(2)$ & $\mathrm{O} 3 \mathrm{~B}-\mathrm{C} 22 \mathrm{~B}-\mathrm{C} 23 \mathrm{~B}-\mathrm{O} 4 \mathrm{~B}$ & $39.4(13)$ \\
\hline
\end{tabular}




\section{supporting information}

$\begin{array}{llll}\mathrm{C} 6 \mathrm{~A}-\mathrm{C} 5 \mathrm{~A}-\mathrm{O} 2 \mathrm{~B}-\mathrm{C} 15 \mathrm{~B} & 103.76(19) & \mathrm{C} 20 \mathrm{~B}-\mathrm{O} 4 \mathrm{~B}-\mathrm{C} 23 \mathrm{~B}-\mathrm{C} 22 \mathrm{~B} & -29.3(12) \\ \mathrm{C} 1 \mathrm{~B}^{\mathrm{ii}}-\mathrm{C} 2 \mathrm{~B}-\mathrm{C} 3 \mathrm{~B}-\mathrm{C} 4 \mathrm{~B}^{\mathrm{ii}} & -3.5(3) & \mathrm{C} 19 \mathrm{~B}-\mathrm{O} 3 \mathrm{~B}-\mathrm{C} 22 \mathrm{C}-\mathrm{C} 23 \mathrm{C} & 11.0(8) \\ \mathrm{C} 7 \mathrm{~B}-\mathrm{C} 2 \mathrm{~B}-\mathrm{C} 3 \mathrm{~B}-\mathrm{C} 4 \mathrm{~B}^{\mathrm{ii}} & 173.31(17) & \mathrm{C} 20 \mathrm{~B}-\mathrm{O} 4 \mathrm{~B}-\mathrm{C} 23 \mathrm{C}-\mathrm{C} 22 \mathrm{C} & 39.4(11) \\ \mathrm{C} 1 \mathrm{~B}^{\mathrm{ii}}-\mathrm{C} 2 \mathrm{~B}-\mathrm{C} 3 \mathrm{~B}-\mathrm{O} 1 \mathrm{~B} & -179.17(15) & \mathrm{O} 3 \mathrm{~B}-\mathrm{C} 22 \mathrm{C}-\mathrm{C} 23 \mathrm{C}-\mathrm{O} 4 \mathrm{~B} & -34.0(13)\end{array}$

Symmetry codes: (i) $-x+1, y,-z+1 / 2$; (ii) $-x+1, y,-z+3 / 2$.

Hydrogen-bond geometry $\left(A,{ }^{\circ}\right)$

$\mathrm{Cg} 1$ is the centroid of the ring $\mathrm{C} 1 B-\mathrm{C} 6 B ; \mathrm{Cg} 2$ is the centroid of the ring $\mathrm{C} 14 A-\mathrm{C} 17 A / \mathrm{N} 1 A / \mathrm{N} 2 A$.

\begin{tabular}{lllll}
\hline$D-\mathrm{H}^{\cdots} \cdots A$ & $D-\mathrm{H}$ & $\mathrm{H} \cdots A$ & $D \cdots A$ & $D-\mathrm{H} \cdots A$ \\
\hline $\mathrm{C} 21 B-\mathrm{H} 21 B \cdots \mathrm{O} 3 A^{\mathrm{iii}}$ & 0.95 & 2.50 & $3.307(3)$ & 143 \\
$\mathrm{C} 18 A-\mathrm{H} 18 A \cdots \mathrm{O} 3 A^{\mathrm{iii}}$ & 0.95 & 2.40 & $3.302(2)$ & 158 \\
$\mathrm{C} 8 B^{\mathrm{iv}}-\mathrm{H} 8 B 2^{\mathrm{iv} \cdots} \mathrm{C} 20 A$ & 0.99 & 2.71 & $3.693(3)$ & 170 \\
$\mathrm{C} 23 A-\mathrm{H} 23 A \cdots \mathrm{Cg} 1^{\mathrm{v}}$ & 0.99 & 2.88 & $3.530(4)$ & 124 \\
$\mathrm{C} 12 B^{\mathrm{vi}}-\mathrm{H} 12 D^{\mathrm{vi} \cdots \mathrm{O} 1 A}$ & 0.99 & 2.71 & $3.414(3)$ & 128 \\
$\mathrm{C} 2 S-\mathrm{H} 2 S \cdots \mathrm{Cg} 2^{\mathrm{i}}$ & 0.95 & 2.67 & $3.609(3)$ & 171
\end{tabular}

Symmetry codes: (i) $-x+1, y,-z+1 / 2$; (iii) $-x+1,-y+2,-z+1$; (iv) $x+1 / 2, y+1 / 2, z$; (v) $-x+3 / 2, y+1 / 2,-z+3 / 2$; (vi) $-x+1,-y+1,-z+1$. 